\title{
TOPOLOGICAL APPLICATIONS OF STANLEY-REISNER RINGS OF SIMPLICIAL COMPLEXES
}

\author{
A. A. AIZENBERG
}

\begin{abstract}
Methods of commutative and homological algebra yield information on the Stanley-Reisner ring $\mathbb{k}[K]$ of a simplicial complex $K$. Consider the following problem: describe topological properties of simplicial complexes with given properties of the ring $\mathbb{k}[K]$. It is known that for a simplicial complex $K=\partial P^{*}$, where $P^{*}$ is a polytope dual to the simple polytope $P$ of dimension $n$, the depth of depth $\mathbb{k}[K]$ equals $n$. A recent construction allows us to associate a simplicial complex $K_{P}$ to any convex polytope $P$. As a consequence, one wants to study the properties of the rings $\mathbb{k}\left[K_{P}\right]$. In this paper, we report on the obtained results for both of these problems. In particular, we characterize the depth of $\mathbb{k}[K]$ in terms of the topology of links in the complex $K$ and prove that depth $\mathbb{k}\left[K_{P}\right]=n$ for all convex polytopes $P$ of dimension $n$. We obtain a number of relations between bigraded betti numbers of the complexes $K_{P}$. We also establish connections between the above questions and the notion of a $k$-Cohen-Macaulay complex, which leads to a new filtration on the set of simplicial complexes.
\end{abstract}

\section{INTRODUCTION}

Cohen-Macaulay rings are classical objects of homological algebra and algebraic geometry. In R. Stanley's monograph 22 and in earlier work of other authors, methods of commutative algebra were used for the study of quotients of polynomial rings by ideals generated by square-free monomials. Reisner 21] noticed that each such ring $R$ is uniquely determined by a simplicial complex $K$, and therefore algebraic properties of $R$ should be studied in terms of the complex $K$. Later on such rings were called Stanley-Reisner rings or face rings. Thus, each simplicial complex gives rise to a graded Stanley-Reisner $\mathbb{k}$-algebra $R=\mathbb{k}[K]$ (see Definition [2.1), where $\mathbb{k}$ is some fixed field.

Stanley-Reisner rings are a very convenient and elegant tool for the study of the combinatorics of simplicial complexes. It is known that a simplicial complex is uniquely determined by its Stanley-Reisner ring [8]. The Hilbert-Poincaré series of the graded algebra $\mathbb{k}[K]$ is given by

$$
\operatorname{Hilb}(\mathbb{k}[K] ; t)=\sum_{i=0}^{n} \frac{f_{i} t^{2 i}}{\left(1-t^{2}\right)^{i}},
$$

where $f_{i}=|\{I \in K:|I|=i\}|$ is the number of simplices of dimension $i-1$ (see [22], [11]). The vector $\left(f_{0}, f_{1}, \ldots, f_{n}\right)$ is called the $f$-vector of $K$. For the study of the complex $K$, it is convenient to use the $h$-vector $\left(h_{0}, \ldots, h_{n}\right)$ defined by the formula $h_{0} t^{n}+\ldots+h_{n-1} t+h_{n}=f_{0}(t-1)^{n}+f_{1}(t-1)^{n-1}+\ldots+f_{n}$. With this definition, the

2010 Mathematics Subject Classification. Primary 13F55; Secondary 55U10, 13 H10.

Key words and phrases. Stanley-Reisner ring, Reisner theorem, depth, Cohen-Macaulay ring, Gorenstein complex, moment-angle complex, nerve-complex.

This work was supported by the grants RFFI 11-01-00694-a and 12-01-92104-YaF_a. 
Hilbert-Poincaré series becomes even simpler:

$$
\operatorname{Hilb}(\mathbb{k}[K] ; t)=\frac{h_{0}+h_{1} t^{2}+\ldots+h_{n} t^{2 n}}{\left(1-t^{2}\right)^{n}} .
$$

This formula allows us to determine properties of $f$ - and $h$-vectors of simplicial complexes from their Stanley-Reisner algebras. One such result is due to Stanley: if $\mathbb{k}[K]$ is a Cohen-Macaulay algebra, then the $h$-vector of $K$ is an $M$-vector (see [18] or 22]). In particular, the $h$-vector of a Cohen-Macaulay complex is nonnegative. Algebraic arguments also show that the Upper Bound Conjecture holds for Cohen-Macaulay complexes: if $K$ is a complex of dimension $n-1$ with $m$ vertices and $\mathbb{k}[K]$ is a Cohen-Macaulay algebra, then the $h$-numbers of $K$ satisfy the inequalities

$$
h_{i}(K) \leqslant\left(\begin{array}{c}
m-n+i-1 \\
i
\end{array}\right), \quad i=0, \ldots,\left[\frac{n}{2}\right] .
$$

Thus, we come to a natural question: what can be said about the complex $K$ whose algebra $\mathbb{k}[K]$ is Cohen-Macaulay? A complete answer to this question is given by Reisner's theorem [21, which says that $\mathbb{k}[K]$ is a Cohen-Macaulay algebra if and only if $\widetilde{H}^{i}\left(\operatorname{link}_{K} I ; \mathbb{k}\right)=0$ for any simplex $I \in K$ and $i<n-1-|I|$. In particular, any simplicial sphere is a Cohen-Macaulay complex. Therefore, inequalities (1.1) hold for simplicial spheres. This observation led to a proof of the long-standing Upper Bound Conjecture for simplicial spheres.

Another important class of commutative rings are Gorenstein* rings (Definition 4.1 in the case of Stanley-Reisner rings). It was shown in [22] that the Stanley-Reisner ring $\mathbb{k}[K]$ is Gorenstein* if and only if $K$ is a generalized homology sphere, i.e., a CohenMacaulay simplicial complex with an extra condition $\widetilde{H}^{n-1-|I|}\left(\operatorname{link}_{K} I ; \mathbb{k}\right)=\mathbb{k}$ for all simplices $I \in K$. For Gorenstein* rings one has important duality theorems. According to a theorem of Avramov-Golod ([2], [9, Th. 3.4.5]), the Tor-algebra $\operatorname{Tor}_{\mathbb{k}[m]}(\mathbb{k}[K], \mathbb{k})$ is a Poincaré algebra if and only if $K=\Delta^{s} * K^{\prime}$ and $K^{\prime}$ is a Gorenstein* complex. From the combinatorial point of view, Gorenstein* complexes are distinguished by the fact that they satisfy the Dehn-Sommerville relations [11]: $h_{i}(K)=h_{n-i}(K)$. From the topological point of view, Gorenstein* complexes are convenient because an analog of the combinatorial Alexander duality holds for them.

Both results, Reisner's theorem and Stanley's theorem characterizing Gorenstein* complexes, are topological, but their original proofs used local cohomology of rings.

In this paper, we consider a filtration on the set of simplicial complexes, unifying the result of [20] and the notion of a $k$-Cohen-Macaulay complex, introduced in [3, 14.

A simplicial complex $K$ is called an $s$-LA-complex over $\mathbb{k}$ if $\widetilde{H}^{i}\left(\operatorname{link}_{K} I ; \mathbb{k}\right)=0$ for any simplex $I \in K$ and $i \leqslant s-1-|I|$. Thus, if $K$ is an $s$-LA-complex, then $K$ is a $t$-LA-complex for $t<s$. Any nonempty simplicial complex is a 0-LA-complex, which follows from the definition. It turns out that the property of being an $s$-LA simplicial complex admits a simple algebraic characterization.

Theorem 1.1. Let $K$ be a simplicial complex and $\mathbb{k}$ a field. The following conditions are equivalent:

(1) $K$ is an $s$-LA-complex;

(2) $\operatorname{depth} \mathbb{k}[K] \geqslant s+1$.

An equivalent formulation of this result can be found in [20, which contains an elegant but somewhat involved proof using homological properties of the cone complex dual to $K$. In Section 3, we offer a simple inductive procedure for proving Theorem 1.1 and other similar results. When $s=\operatorname{dim} K$, Theorem 1.1 implies Reisner's theorem. Thus we have a simple topological proof of Reisner's theorem which does not use local cohomology. 
The combinatorial and topological methods developed in this paper also allow us to prove Stanley's theorem characterizing Gorenstein* complexes (see Section 44).

In Section 5, we investigate connections between the classes of $s$-LA-complexes and the notion of $k$-Cohen-Macaulay complexes (henceforth abbreviated $k$-CM) 3 , 14. The notion of 2-CM was introduced by Richard Stanley as an intermediate property between being Gorenstein and Cohen-Macaulay. Since that time, 2-CM-complexes have been studied in detail in both combinatorics and commutative algebra (see [15, [14], [16] and the references in those papers). Without discussing the algebraic aspects of 2-CMcomplexes (also known as level complexes), we restrict ourselves to some combinatorial properties of general $k$-CM-complexes. It turns out that complexes of types $s$-LA and $k$-CM have similar properties which allow us to introduce a doubly infinite filtration on the set of all simplicial complexes. The properties of this filtration lead to natural proofs of some known results, among which is the following. If $K$ is a Cohen-Macaulay complex of dimension $n-1$, then $K^{(n-2)}$ is a 2-CM-complex [15]. More generally, under the same assumptions, the $n-k$-skeleton $K^{(n-k)}$ is a $k$-CM-complex 14. If $K$ is a 2-CM-complex of dimension $n-1$, then the graph $K^{(1)}$ is $(n-1)$-connected [16. In particular, the last assertion is true for Gorenstein complexes, a result proved under different assumptions in 4 .

One of the main tools used in the proof of Theorem.1.1 is Hochster's formula, which expresses the dimensions of the bigraded components of the Tor-algebra $\operatorname{Tor}_{\mathbb{k}[m]}^{*, *}(\mathbb{k}[K], \mathbb{k})$ in terms of the dimensions of cohomology groups of full subcomplexes of $K$. This formula appeared for the first time in [17. Buchstaber and Panov [1] established a multiplicative isomorphism between the algebra $\operatorname{Tor}_{\mathbb{k}[m]}^{*, *}(\mathbb{k}[K], \mathbb{k})$ and the cohomology algebra of the moment-angle complex $\left(D^{2}, S^{1}\right)^{K}$ associated with the simplicial complex $K$. They posed the problem of describing the multiplicative structure of $\operatorname{Tor}_{\mathbb{k}[m]}^{*, *}(\mathbb{k}[K], \mathbb{k})$ in terms of cohomology of full subcomplexes. A solution was given by Baskakov [5], and that provided a substantial strengthening of Hochster's formula. In Section 6] we recall some of these results.

In Section 7, we use Theorem 1.1 to study spherical nerve-complexes introduced in [1]. To each convex polytope $P$ one canonically associates a simplicial complex $K_{P}$, which is the nerve of the covering of the boundary of $P$ by its facets. If $P$ is a simple polytope, then $K_{P}=\partial P^{*}$ is Cohen-Macaulay, in fact, even Gorenstein*. If $P$ is not simple, then $K_{P}$ is not Cohen-Macaulay, but it still completely determines the combinatorics of $P$. It makes sense to define the Stanley-Reisner algebra of a convex polytope as $\mathbb{k}[P]=\mathbb{k}\left[K_{P}\right]$. In Section 7 we show that $\operatorname{depth} \mathbb{k}[P]=\operatorname{dim} P$ for any convex polytope $P$.

The following question was posed in [1]: what properties distinguish complexes $K_{P}$ in the set of all simplicial complexes? It was this problem that motivated the definition of a spherical nerve-complex. In Section 7 we establish a number of constraints on the bigraded betti numbers of the complexes $K_{P}$, as well as of more general spherical nervecomplexes. It turns out that, for any convex polytope $P$, the simplicial complex $K_{P}$ has properties similar to being Gorenstein* (see Proposition [7.10), but arbitrary spherical nerve-complexes do not necessarily have these properties, as we show in 7.17 .

The bigraded betti numbers of the nerve-complex of a non-simple polytope $P$ yield information on higher-dimensional cohomology of the moment-angle space $\mathcal{Z}_{P}$ associated with the polytope $P$. Namely, we show in Section 7 that $H^{m+n}\left(\mathcal{Z}_{P} ; \mathbb{k}\right)=\mathbb{k}$ and $H^{m+n-1}\left(\mathcal{Z}_{P} ; \mathbb{k}\right)=H^{m+n-2}\left(\mathcal{Z}_{P} ; \mathbb{k}\right)=0$ for any $n$-dimensional polytope $P$ with $m$ facets. Perhaps these relations will lead to a better understanding of the geometry of degenerate intersections of special real quadrics. 
Now we briefly mention basic concepts and notation used in this paper. By definition, a simplicial complex $K$ on the set $[m]=\{1, \ldots, m\}$ is a collection of subsets of $[m]$ such that

(1) $\{i\} \in K$ for all $i \in[m]$;

(2) if $I \in K$ and $J \subset I$, then $J \in K$.

The elements of $K$ are called simplices. The set of vertices of $K$ is sometimes denoted by $V(K)$. For any subset $J \subseteq[m]$, the symbol $|J|$ denotes the cardinality of $J$. In particular, for any simplex $I \in K$ the number $|I|$ is just the number of vertices of the simplex. We have $\operatorname{dim} I=|I|-1$ and $\operatorname{dim} K=\max \{\operatorname{dim} I: I \in K\}$. A simplicial complex $K$ is said to be pure if the dimensions of all maximal with respect to inclusion simplices are the same. If $J \in[m]$, then $\widehat{J}$ will denote the complement: $\widehat{J}=[m] \backslash J$. The symbol $K_{J}$ denotes the full subcomplex of $K$ on the vertices $J \subseteq[m]$, in other words, $K_{J}=\{I \in K: I \subseteq J\}$. The symbol $K^{(s)}$ denotes the $s$-skeleton of $K$, in other words, $K^{(s)}=\{I \in K:|I| \leqslant s+1\}$. The link of a simplex $J \in K$ is the subcomplex $\operatorname{link}_{K} J=\{I \in K: I \cap J=\varnothing, I \cup J \in K\}$; the star of $J$ is $\operatorname{star}_{K} J=\{I \in K: I \cup J \in$ $K\}$. The join of complexes $K_{1}$ and $K_{2}$ is the complex $K_{1} * K_{2}$ on the set $\left[m_{1}\right] \sqcup\left[m_{2}\right]$ whose simplices are of the form $\left\{I_{1} \sqcup I_{2}: I_{1} \in K_{1}, I_{2} \in K_{2}\right\}$. Besides having simple combinatorial descriptions, all these notions also have clear geometric interpretations (see, for example [11, Ch. 2]). The symbol $\widetilde{H}^{i}(X ; \mathbb{k})$ denotes either the module of reduced $i$-dimensional simplicial cohomology of the complex $X$ with coefficients in the ring $\mathbb{k}$ or the module of singular cohomology of the topological space $X$, depending on the context.

\section{A Review of Commutative Algebra}

Henceforth we shall assume that all algebras and modules are graded by integers and are finitely generated. The algebras are (graded) commutative and connected, i.e., $A^{0} \cong \mathbb{k}$.

Let $K$ be a simplicial complex on $m$ vertices with $\operatorname{dim} K=n-1$, and let $\mathbb{k}$ be a field. Some of our results remain true for $\mathbb{k}=\mathbb{Z}$, and such cases will be explicitly mentioned. Let $\mathbb{k}[m]=\mathbb{k}\left[v_{1}, \ldots, v_{m}\right]$ be a graded polynomial algebra in $m$ variables with coefficients in $\mathbb{k}$ and $\operatorname{deg} v_{i}=2$.

Definition 2.1 (The Stanley-Reisner algebra). The Stanley-Reisner algebra is the quotient $\mathbb{k}[m] / I_{\mathrm{SR}}$, where $I_{\mathrm{SR}}$ is an ideal generated by square-free monomials $v_{i_{1}} \ldots v_{i_{k}}$ with $\left\{i_{1}, \ldots, i_{k}\right\} \notin K$.

The canonical projection $p: \mathbb{k}[m] \rightarrow \mathbb{k}[K]$ makes $\mathbb{k}[K]$ a graded $\mathbb{k}[m]$-module.

Definition 2.2. The algebraic dimension (Krull dimension) of a finitely generated graded commutative algebra $A$ is the maximal number of algebraically independent homogeneous elements of $A$. The algebraic dimension of an $A$-module $M$ is the dimension of the algebra $A /$ Ann $M$, where Ann $M=\{a \in A: a M=0\}$.

It is clear that the dimension of $\mathbb{k}[K]$ as an algebra and as a $\mathbb{k}[m]$-module is the same. It is also known [9, 22, that the algebraic dimension of $\mathbb{k}[K]$ equals $\operatorname{dim} K+1=n$.

Definition 2.3. Let $M$ be a module over an algebra $A$. A sequence $\left(t_{1}, \ldots, t_{k}\right)$ of homogeneous elements of positive degrees in $A$ is said to be $M$-regular if $t_{i}$ is not a zerodivisor for the $A$-module $M /\left(t_{1} M+\ldots+t_{i-1} M\right)$ for $i=1, \ldots, k$.

The depth of a module $M$, denoted $\operatorname{depth} M$, is the maximal length of a regular sequence for $M$.

It can be shown that $\mathbb{k}[K]$-regular sequences in $\mathbb{k}[m]$ descend to $\mathbb{k}[K]$-regular sequences in $\mathbb{k}[K]$ and that the sequences lift in the opposite direction. Thus, the depth of $\mathbb{k}[K]$ as 
a $\mathbb{k}[m]$-module and as a $\mathbb{k}[K]$-module is the same. One can also check that the elements of a $\mathbb{k}[K]$-regular sequence in $\mathbb{k}[K]$ are algebraically independent, and therefore

$$
\operatorname{depth} \mathbb{k}[K] \leqslant \operatorname{dim} \mathbb{k}[K] \text {. }
$$

Definition 2.4. An $A$-module $M$ is said to be Cohen-Macaulay if $\operatorname{depth} M=$ $\operatorname{dim} M$. If the algebra $A$ is a Cohen-Macaulay $A$-module, then $A$ is said to be CohenMacaulay.

According to the remark above, the algebra $\mathbb{k}[K]$ is Cohen-Macaulay if and only if it is a Cohen-Macaulay $\mathbb{k}[m]$-module.

Definition 2.5. The simplicial complex $K$ is said to be Cohen-Macaulay over the field $\mathbb{k}$ if $\mathbb{k}[K]$ is a Cohen-Macaulay algebra. $K$ is said to be Cohen-Macaulay over $\mathbb{Z}$ if it is Cohen-Macaulay over the field $\mathbb{Q}$ and over all finite fields.

Now we want to state Reisner's theorem, which motivated the present paper. It describes Cohen-Macaulay simplicial complexes in topological terms.

Theorem 2.6 (Reisner [21). The complex $K$ is Cohen-Macaulay over the field $\mathbb{k}$ if and only if for all simplices $I \in K$ and $i<\operatorname{dim} K-|I|$ we have $\widetilde{H}_{i}\left(\operatorname{link}_{K} I ; \mathbb{k}\right)=0$.

Notice that Reisner's theorem is also true when $\mathbb{k}$ is $\mathbb{Z}$. The proof of this fact reduces to Reisner's theorem for fields by using the universal coefficient theorem. In this case, the homological condition $\widetilde{H}_{j}\left(\operatorname{link}_{K} I ; \mathbb{k}\right)=0$ is equivalent to the cohomological condition $\widetilde{H}^{j}\left(\operatorname{link}_{K} I ; \mathbb{k}\right)=0$. It will be more convenient for us to work with the cohomological versions of the results.

The following reformulation of Reisner's theorem is due to Munkres.

Corollary 2.7 (Munkres, [20, 22, 11]). The complex $K$ is Cohen-Macaulay over $\mathbb{k}$ (a field or $\mathbb{Z})$ if and only if for any point $x$ in the geometric realization $|K|$ of $K$ one has

$$
\widetilde{H}_{i}(|K| ; \mathbb{k})=\widetilde{H}_{i}(|K|,|K| \backslash x ; \mathbb{k})=0
$$

for $i<\operatorname{dim} K$.

By this corollary, the property of a simplicial complex to be Cohen-Macaulay is topological, i.e., it does not depend on the triangulation of $|K|$.

Now we shall list a number of algebraic notions and results which make it easier to work with the depth of a Stanley-Reisner module.

Let $M$ be a finitely generated graded module over a connected finitely generated graded commutative algebra $A$ over the field $\mathbb{k}$. Consider a free resolution

$$
\ldots \rightarrow R^{-i} \rightarrow \ldots \rightarrow R^{-1} \rightarrow R^{0} \rightarrow M \rightarrow 0,
$$

where $R^{-i}$ is a free (or projective) graded module over $A$. Let $N$ be another graded $A$-module. Applying the functor $\otimes_{A} N$ to the chain complex

$$
\ldots \rightarrow R^{-i} \rightarrow \ldots \rightarrow R^{-1} \rightarrow R^{0} \rightarrow 0
$$

we have a chain complex

$$
\ldots \rightarrow R^{-i} \otimes_{A} N \rightarrow \ldots \rightarrow R^{-1} \otimes_{A} N \rightarrow R^{0} \otimes_{A} N \rightarrow 0
$$

of graded $A$-modules. The graded module of the $(-i)$ th cohomology of this complex is denoted by $\operatorname{Tor}_{A}^{-i}(M, N)=\bigoplus_{j} \operatorname{Tor}_{A}^{-i, j}(M, N)$. A standard result in homological algebra says that the bigraded module $\operatorname{Tor}_{A}(M, N)=\bigoplus_{i, j} \operatorname{Tor}_{A}^{-i, j}(M, N)$ does not depend on the choice of the free resolution (2.2).

We are interested in the particular case of this construction when $A=\mathbb{k}[m], M=\mathbb{k}[K]$ is the Stanley-Reisner module, and $N=\mathbb{k}$, where the field $\mathbb{k}$ is viewed as a $\mathbb{k}[m]$-module 
via the surjection $\mathbb{k}[m] \rightarrow \mathbb{k}, v_{i} \mapsto 0$. For the $\mathbb{k}[m]$-module $\mathbb{k}[K]$ we then have the Tor-functor

$$
\operatorname{Tor}_{\mathbb{k}[m]}(\mathbb{k}[K], \mathbb{k})=\bigoplus_{i, j=0}^{m} \operatorname{Tor}_{\mathbb{k}[m]}^{-i, 2 j}(\mathbb{k}[K], \mathbb{k}),
$$

where the degree $2 j$ is induced by the even grading of the Stanley-Reisner module and the degree $-i$ corresponds to the position in the resolution.

Define bigraded betti numbers of $K$ by setting

$$
\beta^{-i, 2 j}(K)=\operatorname{rk}_{\mathbb{k}} \operatorname{Tor}_{\mathbb{k}[m]}^{-i, 2 j}(\mathbb{k}[K], \mathbb{k}), \quad 0 \leqslant i, j \leqslant m .
$$

We also set $\beta^{-i}(K)=\operatorname{rk}_{\mathbb{k}} \operatorname{Tor}_{\mathbb{k}[m]}^{-i, *}(\mathbb{k}[K], \mathbb{k})=\sum_{j} \beta^{-i, 2 j}(K)$.

We then have [11, Lemma 8.13].

Proposition 2.8. Let $K$ be a (nonempty) simplicial complex with $m$ vertices with $\operatorname{dim} K=n-1$. Then:

(1) $\beta^{-i, 2 j}(K)=0$ when $j>m$ or $i>j$;

(2) $\beta^{-i, 2 j}(K)=0$ when $i \geqslant j>0$ or $j-i>n$;

(3) $\beta^{0,2 j}(K)=0$ for $j>0 ; \beta^{0,0}(K)=1$.
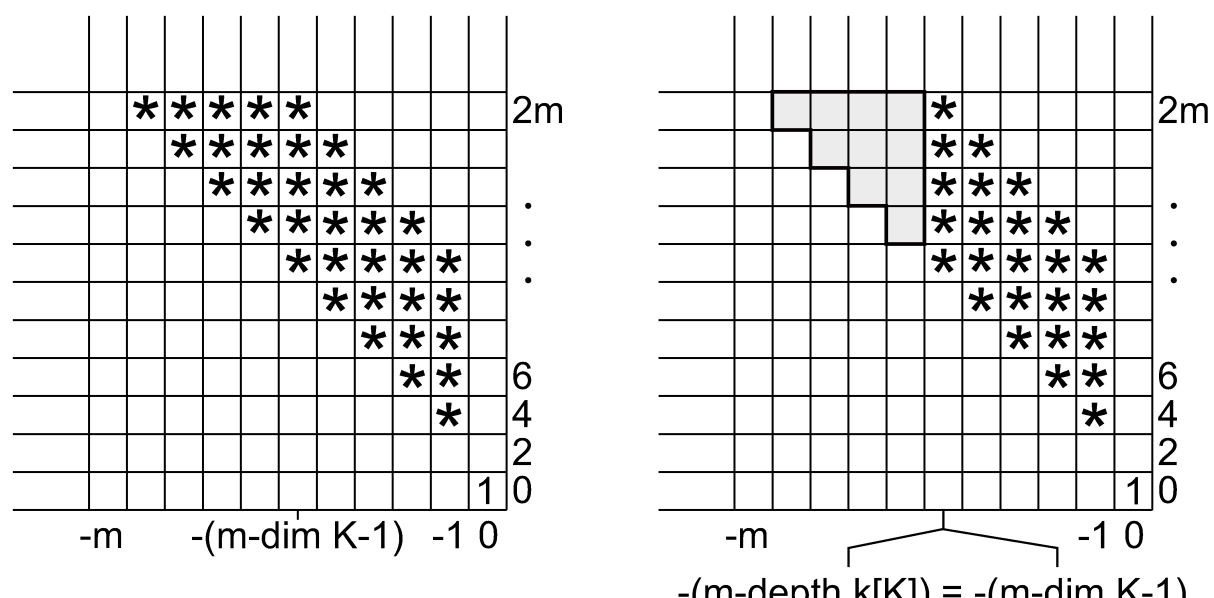

Figure 1. Bigraded betti numbers of an arbitrary complex (left) and of a Cohen-Macaulay complex (right)

Definition 2.9. Let $M$ be an $A$-module. The smallest number $h$ for which there is a free (projective) resolution

$$
\ldots \rightarrow R^{-i} \rightarrow \ldots \rightarrow R^{-1} \rightarrow R^{0} \rightarrow M \rightarrow 0
$$

such that $R^{-i}=0$ for $i>h$ is called the projective dimension of $M$ and is denoted by $\operatorname{pdim} M$.

Examining a minimal resolution (see, for example, [11]) we have $\operatorname{pdim} \mathbb{k}[K]=$ $\max \left\{i: \beta^{-i} \neq 0\right\}$ and

$$
\beta^{-i}(K) \neq 0 \text { for } 0 \leqslant i \leqslant \operatorname{pdim} \mathbb{k}[K] .
$$

Theorem 2.10 (Auslander-Buchsbaum). Let $M \neq 0$ be an A-module with pdim $M$ $<\infty$. Then

$$
\operatorname{pdim} M+\operatorname{depth} M=\operatorname{depth} A .
$$


For a proof of this fact, see [11, Theorem 3.26] or [9, Th.1.3.3]. Notice that depth $\mathbb{k}[m]$ $=m$ because $\left(v_{1}, \ldots, v_{m}\right)$ is a regular sequence of length $m$ in $\mathbb{k}[m]=\mathbb{k}\left[v_{1}, \ldots, v_{m}\right]$, and, therefore, $m \leqslant \operatorname{depth} \mathbb{k}[m] \leqslant \operatorname{dim} \mathbb{k}[m]=m$.

Thus, applying the Auslander-Buchsbaum theorem to the $\mathbb{k}[m]$-module $\mathbb{k}[K]$, we have

$$
\operatorname{pdim} \mathbb{k}[K]+\operatorname{depth} \mathbb{k}[K]=m,
$$

since $\operatorname{pdim} \mathbb{k}[K]<\infty$ by Proposition 2.8 .

Proposition 2.11. Let $K$ be a simplicial complex on $m$ vertices. Then $\operatorname{depth} \mathbb{k}[K]$ $=m-\max \left\{i: \operatorname{Tor}_{\mathbb{k}[m]}^{-i}(\mathbb{k}[K], \mathbb{k}) \neq 0\right\}$. In other words, depth $\mathbb{k}[K] \geqslant s$ if and only if $\beta^{-i}(K)=0$ for $i>m-s$.

Corollary 2.12. Let $K$ be a simplicial complex of dimension $n-1$ on $m$ vertices. Then $K$ is a Cohen-Macaulay complex over $\mathbb{k}$ if and only if $\beta^{-i}(K)=0$ for $i>m-n$.

Remark 2.13. If $K$ is a Cohen-Macaulay complex, then $\beta^{m-n}(K) \neq 0$. Otherwise, we would have $\operatorname{depth} \mathbb{k}[K]>n=\operatorname{dim} \mathbb{k}[K]$, contrary to (2.1).

The positions of the bigraded betti numbers of a Cohen-Macaulay complex are shown in Figure 1 on the right. The numbers in the framed triangle are all zero when the complex is Cohen-Macaulay, unlike the case of an arbitrary simplicial complex.

Now we want to express the conditions for the vanishing of the bigraded betti numbers in topological terms. To this end, we will need Hochster's formula for bigraded betti numbers [17, 22, Theorem 4.8].

Theorem 2.14 (Hochster). Let $K_{J}$ denote the full subcomplex of $K$ on the vertex set $J$. Then

$$
\beta^{-i, 2 j}(\mathbb{k}[K])=\sum_{J \subseteq[m],|J|=j} \operatorname{rk}_{\mathbb{k}} \widetilde{H}^{j-i-1}\left(K_{J} ; \mathbb{k}\right) .
$$

Here we follow the convention that $\widetilde{H}^{-1}(\varnothing ; \mathbb{k})=\mathbb{k}$.

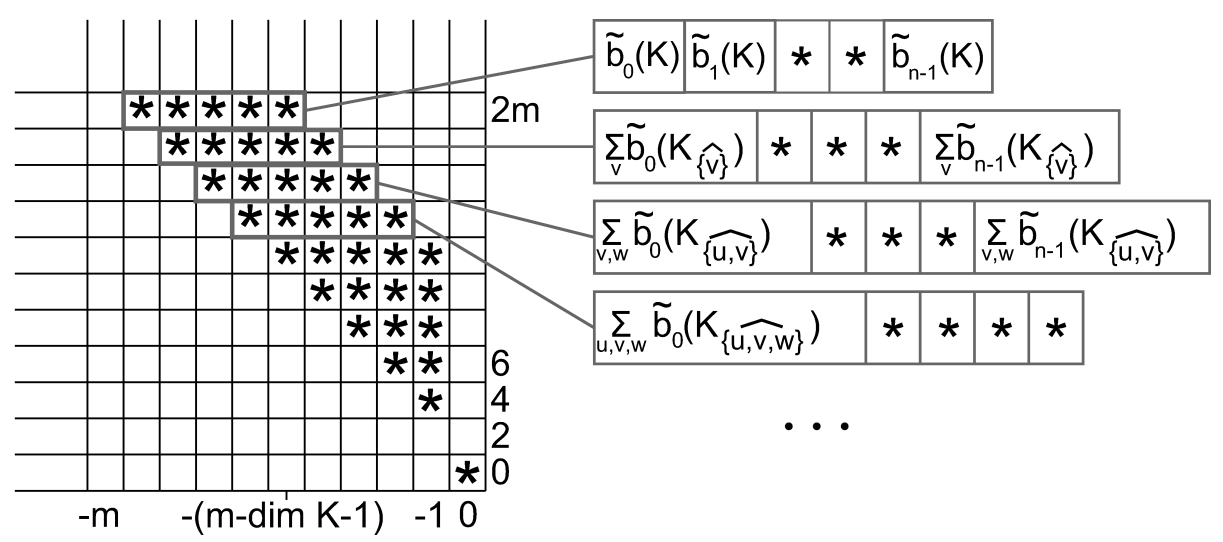

FiguRE 2. Hochster's formula

The first proof of this formula was given in [17, with simpler proofs appearing later in [5, Theorem 1] and [1].

Hochster's formula is schematically shown in Figure 2, Recall that $\widehat{I}=[m] \backslash I$ and $K_{\widehat{I}}$ is the full subcomplex on $\widehat{I}$. Thus, $K_{\widehat{I}}$ is obtained from $K$ by removing all simplices containing at least one vertex from $I$. The symbol $\tilde{b}_{i}$ stands for reduced betti numbers: $\tilde{b}_{i}(X)=\operatorname{rk} \widetilde{H}^{i}(X ; \mathbb{k})$. 
Corollary 2.15. Let $K$ be a simplicial complex on $m$ vertices. Then depth $K \geqslant s$ if and only if $\widetilde{H}^{p}\left(K_{\widehat{J}} ; \mathbb{k}\right)=0$ for all $J \subset[m]$ and $p<s-1-|J|$.

Proof. By Proposition 2.11 and Theorem 2.14, the condition depth $\mathbb{k}[K] \geqslant s$ is equivalent to the following:

$$
\widetilde{H}^{|J|-i-1}\left(K_{J} ; \mathbb{k}\right)=0 \quad \text { for } i>m-s,
$$

or, equivalently, to

$$
\widetilde{H}^{m-|J|-i-1}\left(K_{\widehat{J}} ; \mathbb{k}\right)=0 \quad \text { for } i>m-s .
$$

Setting $p=m-|J|-i-1$, we have $\widetilde{H}^{p}\left(K_{\widehat{J}} ; \mathbb{k}\right)=0$ for $p<m-|J|-m+s-1=s-1-|J|$, as was claimed.

Thus, we have shown that the condition $\operatorname{depth} \mathbb{k}[K] \geqslant s$ is equivalent to a certain acyclicity condition for subcomplexes of $K$. In the next section, we want to show that the acyclicity condition for "punctured" complexes is equivalent to the acyclicity of links. This will lead us to a proof of Theorem 1.1 and, as a consequence, to Reisner's theorem.

\section{Proof of Theorem 1.1}

Definition 3.1. A space (simplicial complex) $L$ is said to be $l$-acyclic (over $\mathbb{k}$ ) if $\widetilde{H}^{i}(L ; \mathbb{k})=0$ for $i \leqslant l$.

Fix an integer $s$. The complex $K$ is said to be link-acyclic of degree $s$ (or an $s$-LAcomplex) if for any simplex $I \in K$ the simplicial complex $\operatorname{link}_{K} I$ is $(s-1-|I|)$-acyclic. In particular, for $I=\varnothing$ we have that $K$ is $(s-1)$-acyclic.

The complex $K$ is said to be subcomplex-acyclic of rank $s$ (or an $s$-SCA-complex) if for any vertex set $J \subseteq[m]$ the simplicial complex $K_{\widehat{J}}$ is $(s-1-|J|)$-acyclic.

Proposition 3.2. Let $\mathbb{k}$ be a field or $\mathbb{Z}$. For any $s \geqslant 0, K$ is an $s$-LA-complex over $\mathbb{k}$ if and only if it is an $s$-SCA-complex over $\mathbb{k}$.

Proof. The next lemma is crucial for the proof of this and of the subsequent results.

Lemma 3.3. Let $\mathbb{k}$ be a field or $\mathbb{Z}, L$ an l-acyclic over $\mathbb{k}$ simplicial complex, and $v$ one of its vertices. Then $\operatorname{link}_{L} v$ is $(l-1)$-acyclic over $\mathbb{k}$ if and only if $L_{\{\widehat{v}\}}$ is $(l-1)$-acyclic over $\mathbb{k}$.

Proof. Consider the subcomplex $\operatorname{star}_{L} v$. Clearly, $L=\left(\operatorname{star}_{L} v\right) \cup L_{\widehat{\{v\}}}$ and, moreover, $\left(\operatorname{star}_{L} v\right) \cap L_{\widehat{\{v\}}}=\operatorname{link}_{L} v$. We want to examine the Mayer-Vietoris sequence for $\operatorname{star}_{L} v$ and $L_{\widehat{\{v\}}}$ (all cohomology groups have coefficients in $\mathbb{k}$ ),

$$
\ldots \leftarrow \widetilde{H}^{i}(L) \leftarrow \widetilde{H}^{i-1}\left(\operatorname{link}_{L} v\right) \leftarrow \widetilde{H}^{i-1}\left(L_{\widehat{\{v\}}}\right) \oplus \widetilde{H}^{i-1}\left(\operatorname{star}_{L} v\right) \leftarrow \widetilde{H}^{i-1}(L) \leftarrow \ldots
$$

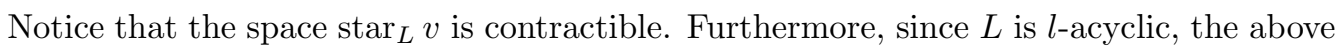
exact sequence for $i \leqslant l$ is of the form

$$
\ldots \longleftarrow \widetilde{H}_{\|}^{i}(L) \longleftarrow \widetilde{H}^{i-1}\left(\operatorname{link}_{L} v\right) \longleftarrow \widetilde{H}^{i-1}\left(L_{\widehat{\{v\}}}\right) \longleftarrow \widetilde{H}_{\|}^{i-1}(L) \longleftarrow \ldots
$$

This implies that $\widetilde{H}^{i-1}\left(\operatorname{link}_{L} v\right)=0 \Leftrightarrow \widetilde{H}^{i-1}\left(L_{\{v\}}\right)=0$ for $i \leqslant l$, as was claimed.

Lemma 3.4. If $K$ is an $s$-LA-complex and $I \in K$, then $\operatorname{link}_{K} I$ is an $(s-|I|)$-LAcomplex.

Proof. This follows directly from the equality $\operatorname{link}_{\operatorname{link}_{K} I} I^{\prime}=\operatorname{link}_{K}\left(I \sqcup I^{\prime}\right)$ for $I^{\prime} \in \operatorname{link}_{K} I$. 
Lemma 3.5. If $K$ is an $s$-SCA-complex and $J \subseteq V(K)$, then $K_{\widehat{J}}$ is an $(s-|J|)$-SCAcomplex.

Proof. This follows from the definition of an SCA-complex.

Now we prove Proposition 3.2 ,

I. First, we show that if $K$ is an $s$-LA-complex, then $K$ is an s-SCA-complex. Call this assertion $P(s)$.

We induct on $s$. For that, we need an auxiliary assertion $L(s)$ : If $K$ is an $s$-LA-complex and $v$ is a vertex of it, then $K_{\widehat{\{v\}}}$ is an $(s-1)$-LA-complex.

The induction will proceed as follows: (1) proofs of $P(0)$ and $L(0),(2)$ deducing $L(s)$ from $L(i)$ for $i<s,(3)$ deducing $P(s)$ from $L(s)$ and $P(s-1)$.

(1) Proof of $P(0)$. By definition, $K$ is a 0 -LA-complex means $\widetilde{H}^{i}\left(\operatorname{link}_{K} I ; \mathbb{k}\right)=0$ for $i \leqslant-|I|-1$. This condition is vacuous when $I \neq \varnothing$, and in the case $I=\varnothing$ it is equivalent to the condition $K \neq \varnothing$.

Similarly, $K$ is a 0 -SCA-complex means that $\widetilde{H}^{i}\left(K_{\widehat{J}} ; \mathbb{k}\right)=0$ for $i \leqslant-|J|-1$. This condition is vacuous when $J \neq \varnothing$, and for $J=\varnothing$ it means that $K \neq \varnothing$. Thus, each of the two conditions 0 -LA and 0-SCA is equivalent to $K$ being nonempty.

Proof of $L(0)$. Let $K$ be a 0 -LA-complex. We have to show that $K_{\widehat{\{v\}}}$ is a $(-1)$ LA-complex. But the condition (-1)-LA is obviously vacuous, and therefore it holds for $K_{\widehat{\{v\}}}$.

(2) Proof of $L(s)$ for $s>0$. Let $K$ be an $s$-LA-complex and $v$ a vertex of it. We shall show that $K_{\widehat{\{v\}}}$ satisfies $(s-1)$-LA. Consider an arbitrary simplex $I \in K_{\widehat{\{v\}}}$. To check that $\operatorname{link}_{K_{\widehat{\{v\}}}} I$ is $(s-|I|-2)$-acyclic, we examine two cases.

Case 1. $I \neq \varnothing$. In this case $\operatorname{link}_{K_{\widehat{\{v\}}}} I=\left(\operatorname{link}_{K} I\right)_{\widehat{\{v\}}}$. By Lemma 3.4 and by the assumptions, $\operatorname{link}_{K} I$ is $(s-|I|)$-LA. Since $|I|>0$ we have, by $L(s-|I|)$, that $\left(\operatorname{link}_{K} I\right)_{\{\widehat{v}\}}$ is $(s-|I|-1)$-LA if $v$ belongs to $\operatorname{link}_{K} I$. If $v \notin \operatorname{link}_{K} I$, then $\left(\operatorname{link}_{K} I\right)_{\widehat{\{v\}}}=\operatorname{link}_{K} I$ is $(s-|I|)$-LA, and therefore it is $(s-|I|-1)$-LA. Thus, $\operatorname{link}_{K_{\widehat{\{v\}}}} I=\left(\operatorname{link}_{K} I\right)_{\widehat{\{v\}}}$ is $(s-|I|-2)$-acyclic, as was claimed.

Case 2. $I=\varnothing$. Now we need to show that $K_{\widehat{\{v\}}}$ is $(s-2)$-acyclic. Notice that, by the assumption, $K$ is $(s-1)$-acyclic and $\operatorname{link}_{K} v$ is $(s-2)$-acyclic. By Lemma $3.3, K_{\widehat{\{v\}}}$ is $(s-2)$-acyclic.

(3) Proof of $P(s)$. Let $K$ be an $s$-LA-complex. Then for any vertex $v \in V(K)$ the complex $K_{\{\widehat{v\}}}$ is $(s-1)$-LA, by the proved assertion $L(s)$. Hence, by the assertion $P(s-1)$, $K_{\widehat{\{v\}}}$ is $(s-1)$-SCA. Therefore, $K_{\widehat{\{v\} \sqcup J}}$ is $(s-1-|J|)$-SCA for any $J \subseteq[m] \backslash\{v\}$. But this is equivalent to saying that $K_{\widehat{J}^{\prime}}$ is $\left(s-\left|J^{\prime}\right|-1\right)$-acyclic for all $J^{\prime} \neq \varnothing$. Together with the equality $\widetilde{H}^{i}(K)=0$ for $i \leqslant s-1$ (which directly follows from the assumed $s$-LA condition for $K)$, this finishes the proof of $P(s)$.

II. Next we show the implication $s$-SCA $\Rightarrow s$-LA.

The outline of the proof is completely analogous to the preceding arguments. Let $Q(s)$ denote the assertion if $K$ is an $s$-SCA-complex, then $K$ is an $s$-LA-complex. Let $M(s)$ denote the assertion if $K$ is an $s$-SCA-complex and $v$ is a vertex of $K$, then $\operatorname{link}_{K} v$ is an $(s-1)$-SCA-complex.

The proof will proceed as follows: (1) proofs of $Q(0)$ and $M(0),(2)$ proof of $M(s)$ using $M(i)$ for $i<s,(3)$ deducing $Q(s)$ from $M(s)$ and $Q(s-1)$.

(1) The assertion $Q(0)$ was proved in I.1. The assertion $M(0)$ says that if $K$ is $0-\mathrm{SCA}$, then $\operatorname{link}_{K} v$ is $(-1)$-SCA. But the condition $(-1)-\mathrm{SCA}$ is vacuous. 
(2) Proof of $M(s)$. Let $K$ be an $s$-SCA-complex. Consider the complex $\operatorname{link}_{K} v$ and let $J$ be an arbitrary set of vertices of $\operatorname{link}_{K} v$. We shall show that the complex $\left(\operatorname{link}_{K} v\right)_{\widehat{J}}$ is $(s-|J|-2)$-acyclic. We have two cases to consider.

Case 1. $J \neq \varnothing$. Then $\left(\operatorname{link}_{K} v\right)_{\widehat{J}}=\operatorname{link}_{K_{\widehat{J}}} v$ and, by Lemma 3.5. $K_{\widehat{J}}$ is $(s-|J|)$-SCA. By the already proved assertion $M(s-|J|), K_{\widehat{J}}$ is $(s-|J|)$-LA. Hence, $\operatorname{link}_{K_{\widehat{J}}} v$ is an $(s-|J|-2)$-acyclic complex.

Case 2. $J=\varnothing$. In this case we need to show that $\operatorname{link}_{K} v$ is $(s-2)$-acyclic. By the assumption, $K$ is $(s-1)$-acyclic and $K_{\widehat{\{v\}}}$ is $(s-2)$-acyclic. By Lemma 3.3, $\operatorname{link}_{K} v$ is $(s-2)$-acyclic, as was claimed.

(3) Proof of $Q(s)$. Let $K$ be an $s$-SCA-complex. By the assumption, $K$ is $(s-1)$ acyclic. Consider an arbitrary nonempty simplex $I^{\prime} \in K$. Then $I^{\prime}=I \sqcup\{v\}$. The simplicial complex $\operatorname{link}_{K} v$ is $(s-1)$-SCA by $M(s)$. By $Q(s-1), \operatorname{link}_{K} v$ is $(s-1)$-LA. Hence, $\operatorname{link}_{\operatorname{link}_{K} v} I=\operatorname{link}_{K} I^{\prime}$ is $(s-1-|I|-1)$-acyclic. Hence, $\operatorname{link}_{K} I^{\prime}$ is $\left(s-1-\left|I^{\prime}\right|\right)$ acyclic, as was claimed.

Theorem 1.1 follows from Proposition 3.2 and Corollary 2.15. Indeed, according to Corollary 2.15, depth $\mathbb{k}[K] \geqslant s+1$ if and only if $K$ is $s$-SCA over the field $\mathbb{k}$. But this is equivalent to the condition $s$-LA over $\mathbb{k}$.

Corollary 3.6 (The Reisner theorem). Let $K$ be a simplicial complex of dimension $n-1$. Then $K$ is Cohen-Macaulay over the field $\mathbb{k}$ if and only if $\widetilde{H}^{i}\left(\operatorname{link}_{K} I ; \mathbb{k}\right)=0$ for all $I \in K$ and $i<n-1-|I|$.

Proof. By definition, $K$ is Cohen-Macaulay if and only if $\operatorname{depth} \mathbb{k}[K]=\operatorname{dim} \mathbb{k}[K]=n$. Taking into account (2.1), this is equivalent to the inequality depth $\mathbb{k}[K] \geqslant n$, which, by Theorem [1.1, is equivalent to the condition $(n-1)$-LA, i.e., $\widetilde{H}^{i}\left(\operatorname{link}_{K} I ; \mathbb{k}\right)=0$ for all $I \in K$ and $i<n-1-|I|$.

The Reisner theorem is often stated in the following form.

Proposition 3.7. $K$ is Cohen-Macaulay over $\mathbb{k}$ if and only if $\widetilde{H}^{i}\left(\operatorname{link}_{K} I ; \mathbb{k}\right)=0$ for

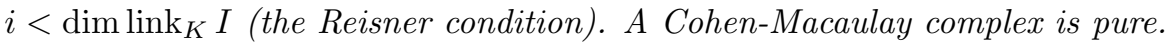

Proof. For $i<\operatorname{dim} K-|I|$, the Reisner condition is equivalent to the equalities $\widetilde{H}^{i}\left(\operatorname{link}_{K} I ; \mathbb{k}\right)=0$. Indeed, the latter implies the former since $\operatorname{dim} \operatorname{link}_{K} I \leqslant \operatorname{dim} K-|I|$. To prove the other implication, we shall show that the Reisner conditions imply that $K$ is pure. Indeed, if $\operatorname{dim} K \geqslant 1$, then $\widetilde{H}^{0}(K ; \mathbb{k})=0$, and therefore $K$ is connected. The links of its vertices are pure complexes (by induction). Furthermore, if two vertices are connected by an edge and their links are pure, then those links have the same dimension. Hence, the dimensions of the links of all vertices are the same, and therefore $K$ is also

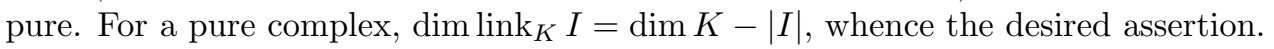

Yet another proof of Proposition 3.7 is contained in the following generalization.

Proposition 3.8. Let $K$ be an $s$-SCA-complex. Then all maximal by inclusion simplices of $K$ have dimension at least $s$.

Proof. $K$ is an $s$-LA-complex by Proposition 3.2 Hence, $\widetilde{H}^{i}\left(\operatorname{link}_{K} I ; \mathbb{k}\right)=0$ for $i<s-|I|$ and, in particular, $\widetilde{H}^{-1}\left(\operatorname{link}_{K} I ; \mathbb{k}\right)=0$ for $|I| \leqslant s$. If $I$ is a maximal by inclusion simplex, then, by definition, $\operatorname{link}_{K} I=\varnothing$, and therefore $\operatorname{rk} \widetilde{H}^{-1}\left(\operatorname{link}_{K} I ; \mathbb{k}\right)=1$. Hence, if $I$ is maximal by inclusion, then $|I| \geqslant s+1$, otherwise the obtained equalities lead to a contradiction. 
If $\operatorname{dim} K=n-1$ and $K$ is Cohen-Macaulay, then $K$ is an $(n-1)$-SCA-complex and all of its maximal simplices have dimension $n-1$, which shows that it is pure. Next, we state a simple geometric consequence of Proposition 3.2

Corollary 3.9. Let $K$ be an $s$-LA-complex and $v_{1}, \ldots, v_{s-1}$ an arbitrary set of its vertices. Then the graph $K_{[m] \backslash\left\{v_{1}, \ldots, v_{s-1}\right\}}^{(1)}$ is connected.

Proof. Proposition 3.2 shows that $K \backslash\left\{v_{1}, \ldots, v_{s-1}\right\}$ is 0 -acyclic, which is equivalent to being connected and nonempty. Hence, its 1-skeleton is also connected.

The Stanley-Munkres theorem, Corollary 2.7, readily admits a generalization to $s$ SCA-complexes, which is due to Munkres [20].

Proposition 3.10. Let $|K|$ be a geometric realization of a simplicial complex $K$. Then $K$ is $s$-SCA $($ over $\mathbb{k})$ if and only if $\widetilde{H}^{i}(|K| ; \mathbb{k})=\widetilde{H}^{i}(|K|,|K| \backslash x ; \mathbb{k})=0$ for any point $x \in|K|$ and $i<s$.

Proof. The proof is completely analogous to that of the Stanley-Munkres theorem (see, for example, [11, Proposition 3.37]). Suppose $x$ lies in the interior of a simplex $I$. Then

$$
\begin{aligned}
\widetilde{H}^{i}(|K|,|K| \backslash x ; \mathbb{k}) & =\widetilde{H}^{i}(\operatorname{star} I, \operatorname{star} I \backslash x ; \mathbb{k}) \\
& =\widetilde{H}^{i}\left(\operatorname{star} I,(\partial I) * \operatorname{link}_{K} I, \mathbb{k}\right) \\
& =\widetilde{H}^{i-1}\left((\partial I) * \operatorname{link}_{K} I ; \mathbb{k}\right) \\
& =\widetilde{H}^{i-|I|}\left(\operatorname{link}_{K} I ; \mathbb{k}\right)
\end{aligned}
$$

by the properties of singular cohomology. Hence $\operatorname{link}_{K} I$ is $(s-|I|-1)$-acyclic if and only if $\widetilde{H}^{i}(|K|,|K| \backslash x ; \mathbb{k})=0$ for $i<s$, which implies the desired assertion.

Thus, the property $s$-LA (which is equivalent to $s$-SCA) of the simplicial complex $K$ is topologically invariant, i.e., it only depends on the topological type of the geometric realization of $|K|$. This implies the main result of 20 .

Proposition 3.11 (Mankres). The depth of the Stanley-Reisner ring is a topological invariant. In other words, if geometric realizations $\left|K_{1}\right|,\left|K_{2}\right|$ of two simplicial complexes are homeomorphic, then $\operatorname{depth} \mathbb{k}\left[K_{1}\right]=\operatorname{depth} \mathbb{k}\left[K_{2}\right]$.

It is convenient to have yet another condition equivalent to $s$-LA and $s$-SCA.

Proposition 3.12. The simplicial complex $K$ is s-SCA if and only if its s-skeleton $K^{(s)}$ is Cohen-Macaulay.

Proof. Notice that $\left(K^{(s)}\right)_{\widehat{J}}=\left(K_{\widehat{J}}\right)^{(s)}$. Then, for $i<s-|J|$ we have $\widetilde{H}^{i}\left(\left(K^{(s)}\right)_{\widehat{J}} ; \mathbb{k}\right)$ $=\widetilde{H}^{i}\left(\left(K_{\widehat{J}}\right)^{(s)} ; \mathbb{k}\right)=\widetilde{H}^{i}\left(K_{\widehat{J}} ; \mathbb{k}\right)=0$, as was claimed.

\section{A characterization of Gorenstein* Complexes}

Another important result from combinatorial commutative algebra is a theorem of Stanley characterizing Gorenstein* simplicial complexes (see [22, 11]). This section contains a topological proof of that theorem.

Let $K$ be a simplicial complex on the set $[m]$. Let

$$
\operatorname{core}[m]=\left\{v \in[m]: \operatorname{star}_{K} v \neq K\right\} .
$$

The core of $K$ is the full subcomplex core $K=K_{\text {core }[m]}$. Thus, $K$ is a join $K=\operatorname{core} K *$ $\Delta^{s-1}$, where $\Delta^{s-1}$ is the simplex on the vertices $[m] \backslash$ core $[m]$. 
Definition 4.1. A Cohen-Macaulay complex $K$ of dimension $n-1$ on $m$ vertices is said to be Gorenstein (over $\mathbb{k})$ if $\operatorname{Tor}_{\mathbb{k}[m]}^{-(m-n)}(\mathbb{k}[K], \mathbb{k}) \cong \mathbb{k}$ or, in other words, $\beta^{-(m-n)}(K)=1$. If, in addition, $K=$ core $K$, then $K$ is said to be Gorenstein* (over $\mathbb{k}$ ).

In this section all complexes are assumed to be Cohen-Macaulay, which means, according to Reisner's theorem, that $\widetilde{H}^{i}\left(\operatorname{link}_{K} I ; \mathbb{k}\right)=0$ for $i \leqslant \operatorname{dim}_{\operatorname{link}} I-1=n-2-|I|$. In particular, $K$ is pure (see Proposition 3.7).

Theorem 4.2 (Stanley). A complex $K$ of dimension $n-1$ is Gorenstein* over the field $\mathbb{k}$ if and only if for any simplex $I \in K$ the complex $\operatorname{link}_{K} I$ has cohomology of the sphere of dimension $\operatorname{dim}_{\operatorname{link}_{K}} I=n-1-|I|$.

The proof will consist of two propositions.

Proposition 4.3. A Cohen-Macaulay complex $K$ of dimension $n-1$ on $m$ vertices is Gorenstein* if and only if

$$
\beta^{-(m-n), 2 m}(K)=1 \text { and } \beta^{-(m-n), 2 j}(K)=0 \text { for } j \neq m .
$$

Proposition 4.4. For a Cohen-Macaulay complex $K$ over $\mathbb{k}$,

$$
\beta^{-(m-n), 2 m}(K)=1 \text { and } \beta^{-(m-n), 2 j}(K)=0 \text { for } j \neq m
$$

if and only if $\widetilde{H}^{n-1-|I|}\left(\operatorname{link}_{K} I ; \mathbb{k}\right)=\mathbb{k}$ for all $I \in K$.

Proof of Proposition 4.3. I. First we show that if

$$
\beta^{-(m-n), 2 m}(K)=1 \text { and } \beta^{-(m-n), 2 j}(K)=0 \text { for } j \neq m,
$$

then $K$ is Gorenstein*. By the assumption, $\beta^{-(m-n)}(K)=1$, and therefore $K$ is Gorenstein by the definition. Suppose $K \neq \operatorname{core} K$. Then $K=\operatorname{core} K * \Delta^{s-1}$, where $s \geqslant 1$. Therefore, $K$ is contractible and, by Hochster's formula, $\beta^{-(m-n), 2 m}(K)=\widetilde{H}^{n-1}(K)=$ 0 , contrary to the assumption.

II. Now we show the other implication: if $K$ is Gorenstein*, then

$$
\beta^{-(m-n), 2 m}=1 \text { and } \beta^{-(m-n), 2 j}(K)=0 \text { for } j \neq m .
$$

In this case, the proof is somewhat more complicated. We begin by setting up notation. Let $L$ be an arbitrary complex on $[m]$ or on a subset of $[m]$. For each $i \in[m]$ define "operators" $p_{i}$ and $q_{i}$ as follows:

$$
\begin{aligned}
& p_{i} L=\operatorname{link}_{L}\{i\}, \\
& q_{i} L=L_{\widehat{\{i\}}},
\end{aligned}
$$

if $i$ is a vertex of $L$. Otherwise, set $q_{i} L=p_{i} L=L$. It is easy to check the following commutation rules in the case $i_{1} \neq i_{2}$ :

$$
\begin{aligned}
& q_{i_{1}} q_{i_{2}} L=q_{i_{2}} q_{i_{1}} L ; \\
& p_{i_{1}} p_{i_{2}} L=\left\{\begin{array}{l}
p_{i_{2}} p_{i_{1}} L=\operatorname{link}_{L}\left\{i_{1}, i_{2}\right\}, \text { if }\left\{i_{1}, i_{2}\right\} \in L, \\
p_{i_{1}} L, \text { or } p_{i_{2}} L, \text { or } L \text { otherwise }
\end{array}\right. \\
& q_{i_{1}} p_{i_{2}} L=\left\{\begin{array}{l}
p_{i_{2}} q_{i_{1}} L, \text { if }\left\{i_{1}, i_{2}\right\} \in L, \\
q_{i_{1}} L, \text { or } p_{i_{2}} L, \text { or } L \text { otherwise; }
\end{array}\right. \\
& p_{i_{1}} q_{i_{2}} L=\left\{\begin{array}{l}
q_{i_{2}} p_{i_{1}} L, \text { if }\left\{i_{1}, i_{2}\right\} \in L, \\
p_{i_{1}} L, \text { or } q_{i_{2}} L, \text { or } L \text { otherwise }
\end{array}\right.
\end{aligned}
$$

We say that complexes $L_{1}$ and $L_{2}$ are $H$-equivalent and write $L_{1} \sim_{H} L_{2}$ (over $\mathbb{k}$ ) if $\widetilde{H}^{i}\left(L_{1} ; \mathbb{k}\right) \cong \widetilde{H}^{i}\left(L_{2} ; \mathbb{k}\right)$ for all $i$. A complex $L$ is said to be acyclic if $L \sim_{H}$ pt, i.e., all its reduced cohomology groups are zero. 
Lemma 4.5. Let $L$ be an acyclic complex on a subset of $[m]$. Then $p_{i} L \sim_{H} q_{i} L$ for any vertex $i \in[m]$.

Proof. If $i$ is not a vertex of $L$, then $p_{i} L=q_{i} L=L$ and we are done. If $i$ is a vertex of $L$, then the desired result follows from the Mayer-Vietoris exact sequence of Lemma 3.3

We now return to the proof of the proposition. By the assumption, $\beta^{-(m-n)}(K)$ $=1$. Suppose $\beta^{-(m-n), 2 m}(K)=0$. Then, by Hochster's formula, there is a set of vertices $J \neq \varnothing$ such that $\widetilde{H}^{n-1-|J|}\left(K_{\widehat{J}} ; \mathbb{k}\right)=\mathbb{k}$ and $\widetilde{H}^{n-1-\left|J^{\prime}\right|}\left(K_{\widehat{J}} ; \mathbb{k}\right)=0$ for any other set $J^{\prime} \neq J$. We shall say that the set $J \neq \varnothing$ is marked. Let $K$ be a complex satisfying the above conditions.

Lemma 4.6. Let $A=\left\{i_{1}, \ldots, i_{k}\right\} \subset[m]$ be any subset of vertices of the complex such that $J \nsubseteq A$. Then $r_{i_{1}} r_{i_{2}} \ldots r_{i_{k}} K$ is acyclic, where $r_{i_{j}}=p_{i_{j}}$ or $q_{i_{j}}$.

Proof. We induct on the number $k$ of elements of $A$. When $k=0$ we have $\widetilde{H}^{i}(K ; \mathbb{k})=0$ for $i<n-1$, as follows from the Cohen-Macaulay condition, and $\widetilde{H}^{n-1}(K ; \mathbb{k})=0$ by the assumption. Hence, $K$ is acyclic and the induction base is proved.

Now assume that $k>0$ is arbitrary. Then $A=\left\{i_{1}\right\} \sqcup A^{\prime}$, where $A^{\prime}=\left\{i_{2}, \ldots, i_{k}\right\}$ also does not contain $J$. Hence, by the induction hypothesis, $r_{i_{2}} \ldots r_{i_{k}} K$ is acyclic for all possible $r_{i_{j}}$. Now apply the commutation rules (4.1). Let $\theta$ be an arbitrary permutation of the indexing set $\left\{i_{1}, \ldots, i_{k}\right\}$. According to the rules, either $r_{i_{1}} r_{i_{2}} \ldots r_{i_{k}} K=$ $r_{\theta\left(i_{1}\right)} r_{\theta\left(i_{2}\right)} \ldots r_{\theta\left(i_{k}\right)} K$ or, at some step of commutation, there was a cancellation and a word on the letters $r_{i_{j}}$ became shorter. In the latter case, $r_{i_{1}} r_{i_{2}} \ldots r_{i_{k}} K=R^{\prime} K$, where the length of $R^{\prime}$ is strictly less than $k$, and therefore, by the induction hypothesis, $R^{\prime} K$ is acyclic. But then all complexes $H$-equivalent to $r_{i_{1}} r_{i_{2}} \ldots r_{i_{k}} K=R^{\prime} K$ are also acyclic. Thus, without loss of generality, we may assume that the variables $r_{i_{j}}$ commute with each other.

Since $r_{i_{2}} \ldots r_{i_{k}} K$ is acyclic by the induction assumption, Lemma 4.5 implies that $p_{i_{1}} r_{i_{2}} \ldots r_{i_{k}} K \sim_{H} q_{i_{1}} r_{i_{2}} \ldots r_{i_{k}} K$. Transposing the variables, we have that the homology type does not change when one of the letters $p_{i_{j}}, q_{i_{j}}$ is replaced by the other in the word $r_{i_{1}} r_{i_{2}} \ldots r_{i_{k}} K$. In particular, this implies that

$$
p_{i_{1}} p_{i_{2}} \ldots p_{i_{k}} K \sim_{H} q_{i_{1}} q_{i_{2}} \ldots q_{i_{k}} K .
$$

Notice that, by definition, $p_{i_{1}} p_{i_{2}} \ldots p_{i_{k}} K=\operatorname{link}_{K}\left\{i_{1}, \ldots, i_{k}\right\}$ and $q_{i_{1}} q_{i_{2}} \ldots q_{i_{k}} K=$ $\left.K_{\left\{i_{1}, \ldots, i_{k}\right.}\right\}$. By the assumption, $\widetilde{H}^{i}\left(K_{\left\{i_{1}, \ldots, i_{k}\right\}} ; \mathbb{k}\right)=0$ for $i=n-1-k$, as well as for $i<n-1-k$, which follows from the Cohen-Macaulay property. On the other hand, by the dimension count, $\widetilde{H}^{i}\left(K_{\left\{i_{1}, \ldots, i_{k}\right\}} ; \mathbb{k}\right)=\widetilde{H}^{i}\left(\operatorname{link}_{K}\left\{i_{1}, \ldots, i_{k}\right\} ; \mathbb{k}\right)=0$ for $i>n-1-k$ since $\operatorname{dim}_{\operatorname{link}_{K}}\left\{i_{1}, \ldots, i_{k}\right\}=n-1-k$. Hence, the simplicial complex $q_{i_{1}} q_{i_{2}} \ldots q_{i_{k}} K$ is acyclic, and the same is true for all complexes $r_{i_{1}} r_{i_{2}} \ldots r_{i_{k}} K$ with arbitrary $r_{i_{j}}$, as was claimed.

Now let $v \in J \neq \varnothing$ be an arbitrary point. If a set $B \subseteq[m]$ contains $v$, then the full subcomplex $K_{B}$ is acyclic. Indeed, the set $A=\left\{i_{1}, \ldots, i_{k}\right\}=\widehat{B}$ does not contain $J$, and therefore, by Lemma 4.6, the complex $q_{A} K=q_{i_{1}} \ldots q_{i_{k}} K=K_{\widehat{A}}=K_{B}$ is acyclic.

Lemma 4.7. Let $v$ be a vertex of the complex $K$ such that for any subset $B \subseteq[m]$ containing $v$, the complex $K_{B}$ is acyclic. Then $K=\operatorname{star}_{K} v$, and therefore $v \notin$ core $K$.

Proof. Assume $I \in K$ and $v \notin I$. We show that $I \sqcup\{v\} \in K$, which would imply the assertion of the lemma. We induct on $|I|$. When $I=\varnothing$ the statement is true. Let $|I|=k$. Then all proper faces of the simplex $I$ also belong to $K$, and, therefore, by the induction

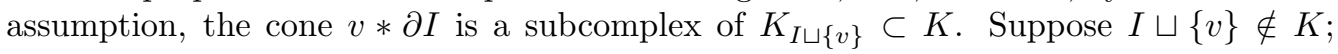


then $K_{I \sqcup\{v\}}=I \cup(v * \partial I)=\partial(I \sqcup\{v\}) \cong S^{|I|-1}$, contrary to the acyclicity of the full subcomplex $K_{I \sqcup\{v\}}$. This shows that $I \sqcup\{v\} \in K$, which proves the induction step.

Thus, there is a vertex $v \in[m]$ such that $\operatorname{star}_{K} v=K$. But this contradicts the fact that $K$ is a Gorenstein* complex. Hence, the assumption $J \neq \varnothing$ is false, and $\beta^{-(m-n), 2 m}(K)=1=\beta^{-(m-n)}(K)$. It now follows that $\beta^{-(m-n), 2 j}(K)=0$ for $j \neq m$. Proposition 4.3 is proved.

Proof of Proposition 4.4. I. First we show that if

$$
\beta^{-(m-n), 2 m}(K)=1 \text { and } \beta^{-(m-n), 2 j}(K)=0 \text { for } j \neq m,
$$

then $\widetilde{H}^{n-1-|I|}\left(\operatorname{link}_{K} I ; \mathbb{k}\right)=\mathbb{k}$ for all simplices $I \in K$. First, notice that

$$
\operatorname{rk} \widetilde{H}^{n-1}(K ; \mathbb{k})=\beta^{-(m-n), 2 m}=1
$$

for $I=\varnothing$. Now we show that $\operatorname{link}_{K} v$ is a Gorenstein* complex. By Proposition 4.3, it suffices to show that

$$
\widetilde{H}^{n-2-|J|}\left(\left(\operatorname{link}_{K} v\right)_{\widehat{J}} ; \mathbb{k}\right)= \begin{cases}\mathbb{k} & \text { if } J=\varnothing, \\ 0 & \text { if } J \neq \varnothing .\end{cases}
$$

Consider first the case $J \neq \varnothing$. Then the exact Mayer-Vietoris sequence of Lemma 3.3

$$
\ldots \longleftarrow \widetilde{H}_{\|}^{n-1-|J|}(K \widehat{J}) \longleftarrow \widetilde{H}^{n-2-|J|}\left(\operatorname{link}_{K_{\widehat{J}}} v\right) \longleftarrow \widetilde{H}^{n-2-|J|}(K \widehat{J \sqcup\{v\}}) \longleftarrow \ldots
$$

for $K_{\widehat{J}}$, shows that $\widetilde{H}^{n-2-|J|}\left(\operatorname{link}_{K_{\widehat{J}}} v ; \mathbb{k}\right)=\widetilde{H}^{n-2-|J|}\left(\left(\operatorname{link}_{K} v\right)_{\widehat{J}}\right)=0$.

In the case $J=\varnothing$, we have

$$
\begin{aligned}
& \widetilde{H}^{n-1}\left(\operatorname{link}_{K} v\right) \longleftarrow \widetilde{H}^{n-1}\left(K_{\{\widehat{v}\}}\right) \longleftarrow \widetilde{H}^{n-1}(K) \longleftarrow \widetilde{H}^{n-2}\left(\operatorname{link}_{K} v\right) \longleftarrow \widetilde{H}^{n-2}\left(K_{\{\widehat{v}\}}\right) \\
& \|\quad\| \quad \|
\end{aligned}
$$

Hence, $\widetilde{H}^{n-2}\left(\operatorname{link}_{K} v ; \mathbb{k}\right)$ is either 0 or $\mathbb{k}$. If $\widetilde{H}^{n-2}\left(\operatorname{link}_{K} v ; \mathbb{k}\right)=0$, then, by what we already proved, $\widetilde{H}^{n-2-|J|}\left(\left(\operatorname{link}_{K} v\right)_{\widehat{J}} ; \mathbb{k}\right)=0$ for any $J$, and therefore, by Hochster's formula, $\beta^{-\left(m^{\prime}-n+1\right), 2 j}\left(\operatorname{link}_{K} v\right)=0$, where $m^{\prime}$ is the number of vertices of $\operatorname{link}_{K} v$. But then, by Proposition 2.11, $\operatorname{depth} \mathbb{k}\left[\operatorname{link}_{K} v\right]>n-1=\operatorname{dim} \mathbb{k}\left[\operatorname{link}_{K} v\right]$, contrary to (2.1). The obtained contradiction shows that $\widetilde{H}^{n-2}\left(\operatorname{link}_{K} v ; \mathbb{k}\right)=\mathbb{k}$.

Thus, we have shown that $\operatorname{link}_{K} v$ is a Gorenstein* complex. By the induction assumption, the links of its simplices are homology spheres. But since $\operatorname{link}_{\operatorname{link}_{K} v} I=$ $\operatorname{link}_{K}(I \sqcup\{v\})$ for all $I \in \operatorname{link}_{K} v$, the links of nonempty simplices of $K$ are also homology spheres. As we mentioned in the beginning of the proof, $K$ itself is a homology sphere.

II. Now we show that if $\widetilde{H}^{n-1-|I|}\left(\operatorname{link}_{K} I ; \mathbb{k}\right)=\mathbb{k}$ for all $I \in K$ and $K$ is a CohenMacaulay complex, then $\beta^{-(m-n), 2 m}(K)=1$ and $\beta^{-(m-n), 2 j}(K)=0$ for $j \neq m$. We induct on $\operatorname{dim} K$. In fact, we shall prove a stronger statement.

Lemma 4.8. Let $K$ be a pure simplicial complex of dimension $n-1$ such that

$$
\widetilde{H}^{j}\left(\operatorname{link}_{K} I ; \mathbb{k}\right)= \begin{cases}\mathbb{k} & \text { if } j=n-1-|I|, \\ 0 & \text { if } j<n-1-|I| .\end{cases}
$$

Then $K$ is Gorenstein* and, in addition, $\widetilde{H}^{n-1}\left(K_{J} ; \mathbb{k}\right)=0$ for any subset $J \neq[m]$. 
Proof. By the assumption, $K$ is a Cohen-Macaulay complex.

It is straightforward to establish the induction base $n-1=0$. Now we prove the assertion for an arbitrary dimension $\operatorname{dim} K=n-1$. Let $v$ be a vertex of $K$. Then, by the assumption, $\operatorname{link}_{K} v$ and the links of all of its simplices are also homology spheres. Hence, by the induction assumption, $\operatorname{link}_{K} v$ is Gorenstein*, and $\widetilde{H}^{n-2}\left(\left(\operatorname{link}_{K} v\right)_{J} ; \mathbb{k}\right)=0$ if $J$ is not the set of vertices of $\operatorname{link}_{K} v$.

(1) Let us prove, by inner induction on $|J|$, that $\widetilde{H}^{n-1}\left(K_{J} ; \mathbb{k}\right)=0$ for $J \neq[m]$. The induction base is obvious because of the dimension considerations: $\widetilde{H}^{n-1}\left(K_{J} ; \mathbb{k}\right)$ $=0$ for $|J|<n$. Now let $J \subset[m]$ be an arbitrary set of vertices, distinct from $[m]$. If $\operatorname{dim} K=0$, then the assertion is proved. Thus, we may assume that $\operatorname{dim} K>0$ and $K$ is a connected complex (because $\widetilde{H}^{0}(K ; \mathbb{k})=0$ when $K$ is Macaulay). Then there is a vertex $v \in J$ such that $\operatorname{star}_{K} v \nsubseteq K_{J}$. Let $J=\{v\} \sqcup J^{\prime}$; then $\left(\operatorname{link}_{K} v\right)_{J^{\prime}} \neq \operatorname{link}_{K} v$, and therefore $\widetilde{H}^{n-2}\left(\left(\operatorname{link}_{K} v\right)_{J^{\prime}} ; \mathbb{k}\right)=0$ by the assumption of outer induction. Consider the exact sequence

$$
\widetilde{H}_{\|}^{n-1}\left(K_{J^{\prime}}\right) \longleftarrow \widetilde{H}^{n-1}\left(K_{J^{\prime} \sqcup\{v\}}\right) \longleftarrow \widetilde{H}^{n-2}\left(\left(\operatorname{link}_{K} v\right)_{J^{\prime}}\right)
$$

of Lemma 3.3. Here, $\widetilde{H}^{n-1}\left(K_{J^{\prime}} ; \mathbb{k}\right)=0$ by the assumption of the inner induction as $\left|J^{\prime}\right|=|J|-1$. Hence, $\widetilde{H}^{n-1}\left(K_{J} ; \mathbb{k}\right)=0$, which is the second part of the assertion.

(2) Let us prove that $K$ is Gorenstein*. By the assumption, $\widetilde{H}^{n-1}(K ; \mathbb{k})=\mathbb{k}$, and therefore we only need to prove that

$$
\widetilde{H}^{n-1-|J|}\left(K_{\widehat{J}} ; \mathbb{k}\right)=0
$$

when $J \neq \varnothing$. First, we prove this for $J=\{v\}$. In this case we have an exact sequence

$$
\widetilde{H}^{n-1}\left(K_{\{\widehat{v v\}}}\right) \longleftarrow \widetilde{H}^{n-1}(K) \longleftarrow \widetilde{H}^{n-2}\left(\operatorname{link}_{K} v\right) \longleftarrow \widetilde{H}^{n-2}\left(K_{\{v\}}\right) \longleftarrow \widetilde{H}^{n-2}(K)
$$

Here $\widetilde{H}^{n-2}(K ; \mathbb{k})=0$, since $K$ is a Cohen-Macaulay complex, $\widetilde{H}^{n-1}(K ; \mathbb{k})$ $=\widetilde{H}^{n-2}\left(\operatorname{link}_{K} v ; \mathbb{k}\right)=\mathbb{k}$ by the assumption, and $\widetilde{H}^{n-1}\left(K_{\widehat{\{v\}}} ; \mathbb{k}\right)=0$ by what we proved in step (1). Hence, $\widetilde{H}^{n-2}\left(K_{\widehat{\{v\}}} ; \mathbb{k}\right)=0$.

Now we prove (4.2) in the case $|J| \geqslant 2$. We have $J=J^{\prime} \sqcup\{v\}$. Consider the exact sequence

$$
\widetilde{H}^{n-1-|J|}\left(\operatorname{link}_{K_{\widehat{J}^{\prime}}} v\right) \longleftarrow \widetilde{H}^{n-1-|J|}\left(K \widehat{J^{\prime} \sqcup\{v\}}\right) \longleftarrow \widetilde{H}^{n-1-|J|}\left(K \widehat{J}^{\prime}\right)
$$

where $\widetilde{H}^{n-1-|J|}\left(K_{\widehat{J}} ; \mathbb{k}\right)=\widetilde{H}^{n-2-\left|J^{\prime}\right|}\left(K_{\widehat{J^{\prime}}} ; \mathbb{k}\right)=0$ by the Cohen-Macaulay assumption. Let $S$ be the set of vertices of $\operatorname{link}_{K} v$ and $T=J^{\prime} \cap S$. Then $\left(\operatorname{link}_{K} v\right)_{\widehat{J}^{\prime}}=\left(\operatorname{link}_{K} v\right)_{\widehat{T}}$ and $|T| \leqslant\left|J^{\prime}\right|$. Then, since $\operatorname{link}_{K} v$ is Gorenstein* by the induction assumption, we have $\widetilde{H}^{n-1-|J|}\left(\operatorname{link}_{K_{\widehat{J}}} v\right)=\widetilde{H}^{n-2-\left|J^{\prime}\right|}\left(\left(\operatorname{link}_{K} v\right)_{\widehat{T}}\right)=0$. The exact sequence (4.3) shows that $\widetilde{H}^{n-1-|J|}\left(K_{\widehat{J^{\prime} \sqcup v}}\right)=\widetilde{H}^{n-1-|J|}\left(K_{\widehat{J}}\right)=0$, and $K$ is Gorenstein*. The lemma is proved.

Lemma 4.8 completes the proof of the assertion.

The results of Proposition 4.3 and Lemma 4.8 are shown in Figure 3. Notice also that, by the Avramov-Golod theorem, the bigraded algebra $\operatorname{Tor}_{\mathbb{k}[m]}^{*, *}(\mathbb{k}[K], \mathbb{k})$ is Poincaré if $K$ is a Gorenstein* complex. Thus, the distribution of the bigraded numbers in Figure 3 is symmetric with respect to the center of the parallelogram. In [10, this duality was 


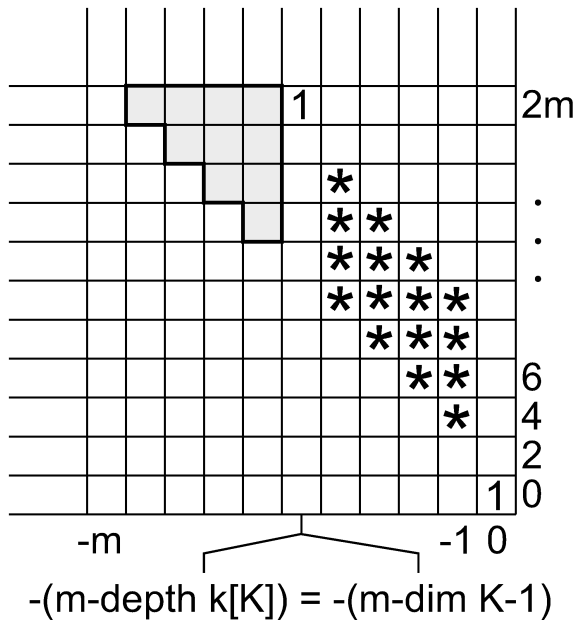

Figure 3. Bigraded betti numbers of a Gorenstein* complex

deduced from the Poincaré duality for the moment-angle manifold of $K$ when $K$ was a sphere. We will discuss this result in more detail in Section 6 .

\section{The $k$-Cohen-Macaulay Condition and the EXtended Filtration OF THE SET OF SIMPLICIAL COMPLEXES}

This section contains a survey of the theory of $k$-Cohen-Macaulay complexes and its connections with the results from the previous sections. This theory appeared and was developed in [3, 15, 14] and other papers.

Definition 5.1. Let $k \in \mathbb{N}$ be a fixed number. A simplicial complex $K$ of dimension $n-1$ on $m$ vertices is said to be $k$-Cohen-Macaulay if $K_{\widehat{J}}$ is Cohen-Macaulay of dimension $n-1$ for all $J \subseteq[m]$ such that $|J|<k$.

In particular, if $K$ is $k$-Cohen-Macaulay ( $k$-CM for short), then it is $l$-CM for $l<k$. The condition 1-CM is equivalent to simply being Cohen-Macaulay.

Example 5.2. Notice that the simplex $K=\Delta^{n-1}$ is Cohen-Macaulay but not $k$-CM for $k>1$ since $\operatorname{dim} K_{\widehat{J}}<\operatorname{dim} K$ for $J \neq \varnothing$.

The following assertion is known to experts in commutative algebra (see the introduction to [15] and the references there). 2-CM-complexes are also known as level complexes.

Proposition 5.3. Let $K$ be a Gorenstein* complex. Then $K$ is 2-CM.

Proof. Let $\operatorname{dim} K=n-1$. Then, by the definition of a Gorenstein* complex and Hochster's formula, for any vertex $v$ and subset $J \subseteq[m] \backslash\{v\}$ we have $\widetilde{H}^{i}\left(\left(K_{\widehat{v}}\right)_{\widehat{J}} ; \mathbb{k}\right)=$ 0 for $i \leqslant n-1-|J \sqcup\{v\}|$. Hence, $K_{\widehat{v}}$ is $(n-1)$-SCA. By Theorem 1.1, we have $\operatorname{depth} \mathbb{k}\left[K_{\widehat{v}}\right] \geqslant n$. Since

$$
n \leqslant \operatorname{depth} \mathbb{k}\left[K_{\widehat{v}}\right] \leqslant \operatorname{dim} K_{\widehat{v}}+1 \leqslant \operatorname{dim} K+1=n,
$$

we have $\operatorname{dim} K_{\widehat{v}}+1=\operatorname{depth} \mathbb{k}\left[K_{\widehat{v}}\right]=n$. Hence, $K_{\widehat{v}}$ is Cohen-Macaulay of the same dimension as $K$. By definition, this means that $K$ is 2-CM-complex.

By Hochster's formula, the bigraded betti numbers of a $k$-CM-complex look like those in Figure 4. The numbers in the shaded parallelogram are zero. 


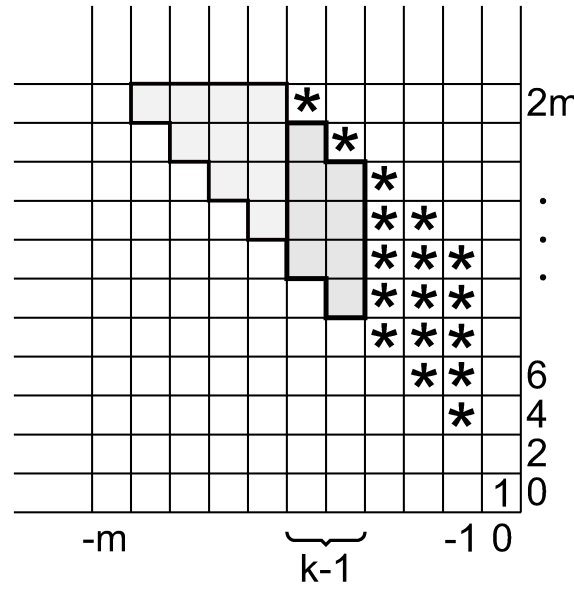

FiguRE 4. Bigraded betti numbers of a $k$-CM-complex

The 1-skeleton of a $k$-CM-complex has a higher order of connectivity than the 1skeleton of a Cohen-Macaulay complex. If $K$ is a Cohen-Macaulay complex of dimension $n-1$, then, by Corollary 3.9 , the graph $K^{(1)}$ is $(n-1)$-connected. In other words, for any set of $n-2$ vertices $\left\{v_{1}, \ldots, v_{n-2}\right\} \subseteq[m]$, the graph $K_{\left\{v_{1}, \widehat{(1)}, \widehat{v_{n}-2}\right\}}$ is connected.

Proposition 5.4. Let $K$ be $k$-CM-complex of dimension $n-1$. Then the graph $K^{(1)}$ is $(n+k-2)$-connected. In other words, $K^{(1)}$ remains connected after removal of any $n+k-3$ vertices.

Proof. Let $J \subseteq[m]$ be an arbitrary set of $|J| \leqslant k-1$ vertices. Then $K_{\widehat{J}}$ is a CohenMacaulay complex of dimension $n-1$ and Corollary 3.9 applies, according to which the graph $\left(K_{\widehat{J}}^{(1)}\right)_{\left\{v_{1}, \widehat{, \ldots, v_{n}-2}\right\}}$ is connected. Hence, for any set of vertices $I=J \sqcup\left\{v_{1}, \ldots, v_{n-2}\right\}$ of cardinality at most $k-1+n-2$, the graph $K_{\widehat{I}}^{(1)}$ is connected.

In particular, the 1-skeleton of any 2-CM-complex $K$ of dimension $n-1$ is $n$-connected (in the sense of graph theory). Taking into account Proposition 5.3, we have the following result [4].

Corollary 5.5 (4], [16]). The 1-skeleton of a Gorenstein * complex $K$ of dimension $n-1$ is an n-connected graph.

A proof using properties of bigraded betti numbers of the Stanley-Reisner ring, which is similar to ours, can be found in [16].

In particular, we have that any simplicial sphere of dimension $n-1$ has an $n$-connected 1-skeleton. Now we want to state some technical lemmas needed to handle $l$-SCA- and $k$-CM-complexes.

Lemma 5.6. Let $K$ be an $l$-SCA-complex. Then $K^{(i)}$ is also l-SCA for $i \geqslant l$.

The proof is similar to that of Proposition 3.12, which is a particular case of this result with $i=l$.

Lemma 5.7. Let $K$ be an l-SCA-complex. Then $K^{(i)}$ is a Cohen-Macaulay complex for $i \leqslant l$.

Proof. We shall show that $\widetilde{H}^{s}\left(K_{\widehat{J}}^{(i)} ; \mathbb{k}\right)=0$ for all $J \subseteq[m]$ and $s<i-|J|$. We have $s<i-|J| \leqslant l-|J|$. Since $s<i, \widetilde{H}^{s}\left(K_{\widehat{J}}^{(i)} ; \mathbb{k}\right)=\widetilde{H}^{s}\left(K_{\widehat{J}} ; \mathbb{k}\right)$. But $\widetilde{H}^{s}\left(K_{\widehat{J}} ; \mathbb{k}\right)=0$ for 
$s<l-|J|$ since $K$ is $l$-SCA. Hence, $\widetilde{H}^{s}\left(K_{\widehat{J}}^{(i)} ; \mathbb{k}\right)=0$ for $s<i-|J|$. Therefore, $K^{(i)}$ is an $i$-SCA-complex of dimension $i$, i.e., $K^{(i)}$ is a Cohen-Macaulay complex.

Proposition 5.8. Let $K$ be a Cohen-Macaulay complex of dimension $n-1$, and $t \leqslant n-1$. Then the skeleton $K^{(t)}$ is an $(n-t)$-CM-complex.

Proof. Let $J$ be a set of vertices $K$ with $|J|<n-t$. By the assumption, $K$ is $(n-1)$-SCA. By Lemma 3.5, $K_{\widehat{J}}$ is $(n-|J|-1)$-SCA. Since $n-|J|-1>n-(n-t)-1=t-1$, i.e., $t \leqslant n-|J|-1$, Lemma 5.7 shows that $K_{\widehat{J}}^{(t)}$ is a Cohen-Macaulay complex of dimension $t$. But this condition being true for all $J$ such that $|J|<n-t$ means precisely that $K^{(t)}$ is an $(n-t)$-CM-complex.

In particular, it follows from Proposition 5.8 that if $K$ is a Cohen-Macaulay complex of dimension $n-1$, then $K^{(n-2)}$ is 2-CM. This result was also obtained in [15]. A more general statement was formulated in [14:

Proposition 5.9. Let $K$ be a $k$-CM-complex of dimension $n-1$. Then $K^{(t)}$ is $(k+n-$ $1-t)-\mathrm{CM}$ for $t \leqslant n-1$.

Proof. By the assumption, $K_{\widehat{J}}$ is Cohen-Macaulay for $|J|<k$. By Proposition [5.8, $K_{\widehat{J}}^{(t)}$ is $(n-t)$-CM. By definition, $\left(K_{\widehat{J}}^{(t)}\right)_{\widehat{I}}=\left(K^{(t)}\right)_{\widehat{J} \sqcup I}$ is Cohen-Macaulay of dimension $t$ for $|J|<k,|I|<n-t$. Hence, $\left(K^{(t)}\right)_{\widehat{J \sqcup I}}$ is Cohen-Macaulay of dimension $t$ for $|J \sqcup I| \leqslant k-1+n-t-1$. Therefore, $K^{(t)}$ is a $(k+n-1-t)$-CM-complex.

It is convenient to have the following generalization of Proposition 5.8 .

Proposition 5.10. Let $K$ be an l-SCA-complex and $i \leqslant l$. Then $K^{(i)} i s(l-i+1)$-CM.

Proof. By Proposition 3.12, $K^{(l)}$ is a Cohen-Macaulay complex of dimension $l$. Applying Proposition 5.8, we have the desired assertion.

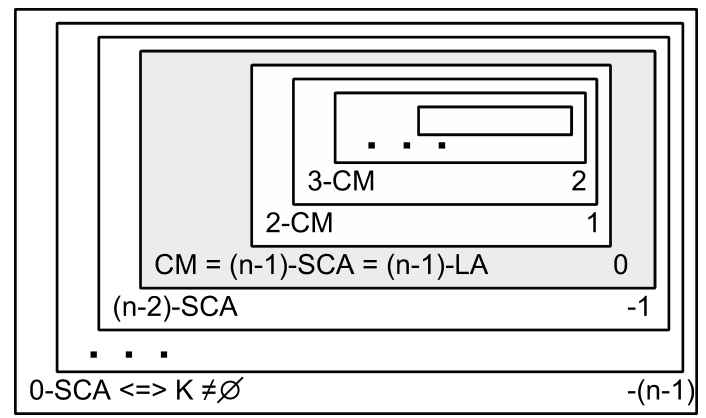

FiguRE 5. Filtration on the set of simplicial complexes

To summarize the foregoing discussion, we consider a doubly-infinite filtration on the set of simplicial complexes. Fix the dimension of complexes $\operatorname{dim} K=n-1$. We say that the complex $K$ of dimension $n-1$ belongs to the class $\mathcal{A}_{i}^{n-1}$ if either $i \geqslant 0$ and $K$ is $(i+1)$-CM or $i<0$ and $K$ is $(n-1+i)$-SCA. We have the following chain of inclusions:

$$
\ldots \supset \mathcal{A}_{-2}^{n-1} \supset \mathcal{A}_{-1}^{n-1} \supset \mathcal{A}_{0}^{n-1} \supset \mathcal{A}_{1}^{n-1} \supset \mathcal{A}_{2}^{n-1} \supset \ldots
$$

Notice that $\mathcal{A}_{-n}^{n-1}=\mathcal{A}_{-n-1}^{n-1}=\ldots$ and that this class coincides with the set of all simplicial complexes of dimension $n-1$ since the condition $l$-SCA is vacuous for $l<0$. Set $\mathcal{A}_{i}=\bigcup_{n} \mathcal{A}_{i}^{n-1}$. 
Theorem 5.11. (1) Let $K \in \mathcal{A}_{i}^{n}$. Then $K^{(l)} \in \mathcal{A}_{i+n-l}^{l}$ for $l \leqslant n$.

(2) Let $K \in \mathcal{A}_{i}$ and let $J$ be a subset of vertices of $K$. Then $K_{\widehat{J}} \in \mathcal{A}_{i-|J|}$.

(3) Let $K \in \mathcal{A}_{i}$ and $I \in K$. Then $\operatorname{link}_{K} I \in \mathcal{A}_{i}$.

Proof. Assertion (1) follows from Lemma 5.6] and Propositions 5.9 and 5.10, Notice that it suffices to prove (2) and (3) for $|J|=|I|=1$. For $i>0$, (2) follows from the definition of a $k$-CM-complex, and for $i \leqslant 0$ from the definition of an SCA-complex.

Now we prove (3). Let $\operatorname{dim} K=n-1$ and $I=\{v\}$. Suppose first that $i \leqslant 0$. Then, by the assumption, $K$ is $(n-1+i)$-LA. By Lemma 3.4 $\operatorname{link}_{K} v$ is $(n-2+i)$-LA. Hence, $\operatorname{link}_{K} v \in \mathcal{A}_{n-2+i-\operatorname{dim}_{\text {link }} v}$, and therefore $\operatorname{link}_{K} v \in \mathcal{A}_{i}$, because $\operatorname{dim} \operatorname{link}_{K} v \leqslant n-2$.

Suppose that $i>0$ (see also [14, Lemma 3.4]). In this case, $K$ is $(i+1)-C M$, and we need to show that the link of any of its vertices has the same property. Let $I^{\prime}$ be a set of vertices of $\operatorname{link}_{K} v$ such that $\left|I^{\prime}\right| \leqslant i$. Notice that $K_{\widehat{I}^{\prime}}$ is Cohen-Macaulay of dimension $n-1$, because $K$ is $(i+1)$-CM. But then $\left(\operatorname{link}_{K} v\right)_{\widehat{I}^{\prime}}=\operatorname{link}_{K_{\widehat{I}^{\prime}}} v$ is Cohen-Macaulay of dimension $n-2$, which was claimed.

Thus, we have a rule: when passing to a skeleton of a smaller dimension the class of the complex increases, when vertices are removed the class of the complex becomes smaller, and when passing to a link the class of the complex remains the same.

Now we want to briefly discuss topological invariance properties of the filtration (5.1). When $i \leqslant 0$ the condition $K \in \mathcal{A}_{i}$ is topologically invariant by Proposition [3.10. The condition $K \in \mathcal{A}_{1}$ (i.e., $K$ is a 2 -CM-complex) is also topologically invariant (see the introduction in [19]). However, when $i>1$, the topological invariance may no longer hold, as can be seen from the following example.

Example 5.12. Let $K=K_{4}=\left(\Delta^{3}\right)^{(1)}$ be a full graph on four vertices. Then, by Proposition 5.8, $K$ is 3-CM, since $\Delta^{3}$ is Cohen-Macaulay. On the other hand, it is easy to check that the complex $K^{\prime}$, obtained from $K$ by a barycentric subdivision of one of its edges, is no longer 3-CM.

We conclude the discussion of $k$-CM-complexes by the following proposition, which can be proved using Figures 3 and 4 .

Proposition 5.13. The classes of Gorenstein* complexes and 3-CM-complexes do not intersect.

Proof. Suppose there is a simplicial complex $K$ of dimension $n-1$ on $m$ vertices which is both Gorenstein* and 3-CM. Then, by Lemma 4.8, $\widetilde{H}^{n-1}\left(K_{\widehat{\{v\}}}\right)=0$ for any vertex $v$. Hence, by Hochster's formula, $\beta^{-(m-n-1), 2(m-1)}(K)=0$. On the other hand, the condition 3-CM implies that for any pair of vertices $\left\{v_{1}, v_{2}\right\}$ the complex $K_{\left\{\widehat{v_{1}, v_{2}}\right\}}$ is Cohen-Macaulay of dimension $n-1$, and, in particular, $\widetilde{H}^{n-2-|J|}\left(K_{\left\{v_{1}, v_{2}\right\}} \sqcup J\right) \stackrel{\left\{v_{1}, v_{2}\right\}}{=}$ for any subset $J \subseteq[m] \backslash\left\{v_{1}, v_{2}\right\}$. By Hochster's formula, we have

$$
\beta^{-(m-n-1), 2(m-2-j)}(K)=0
$$

for all all $j \geqslant 0$. Hence, $\beta^{-(m-n-1)}(K)=0$ but $\beta^{-(m-n)}(K) \neq 0$. This contradicts (2.5), which holds for all simplicial complexes.

\section{Moment-Angle SPACE AND NeRVE-COMPleXes of POlytopes}

This section contains a survey of the topological interpretation of the bigraded betti numbers of simplicial complexes, as defined in [5], 6], and [10].

Fix a two-dimensional disk $D^{2}=\{z \in \mathbb{C}:|z| \leqslant 1\}$. Let $K$ be a simplicial complex on $m$ vertices. For each simplex $I \in K$ consider the subset $U_{I}$ of the polydisk $\left(D^{2}\right)^{m}$ defined by the following condition: $\left(z_{1}, z_{2}, \ldots, z_{m}\right) \in U_{I}$ if and only if $\left|z_{i}\right|=1$ for all 
$i \notin I$. In other words, $U_{I}=\left(D^{2}\right)^{I} \times\left(S^{1}\right)^{[m] \backslash I}$, where $S^{1}=\partial D^{2}=\{z \in \mathbb{C}:|z|=1\}$. One can see that any cellular structure on the pair $\left(D^{2}, S^{1}\right)$ gives rise to a canonical cellular decomposition of $U_{I}$.

Definition 6.1. The space $\left(D^{2}, S^{1}\right)^{K}=\bigcup_{I \in K} U_{I} \subseteq\left(D^{2}\right)^{m}$ is called the moment-angle complex of $K$.

Example 6.2. Let $K=\partial \Delta^{2}$ be the complex on the set of three vertices with maximal simplices $\{1,2\},\{1,3\},\{2,3\}$. Then $\left(D^{2}, S^{1}\right)^{K}=D^{2} \times D^{2} \times S^{1} \cup D^{2} \times S^{1} \times D^{2} \cup S^{1} \times$ $D^{2} \times D^{2}=\partial\left(D^{2} \times D^{2} \times D^{2}\right) \cong S^{5}$. Similarly $\left(D^{2}, S^{1}\right)^{\partial \Delta^{k}} \cong S^{2 k+1}$.

To describe the cohomology ring of $\left(D^{2}, S^{1}\right)^{K}$ over $\mathbb{k}$ (a field or the ring $\mathbb{Z}$ ), one uses the bigraded differential algebra

$$
R^{*}(K)=\Lambda\left[u_{1}, \ldots, u_{m}\right] \otimes \mathbb{k}[K] /\left(v_{i}^{2}=u_{i} v_{i}=0, i=1, \ldots, m\right),
$$

where bideg $u_{i}=(-1,2)$, bideg $v_{i}=(0,2), d u_{i}=v_{i}, d v_{i}=0$. Here $\Lambda\left[u_{1}, \ldots, u_{m}\right]$ is the exterior algebra over $\mathbb{k}$ and $\mathbb{k}[K]=\mathbb{k}\left[v_{1}, \ldots, v_{m}\right] / I_{\mathrm{SR}}$ is the Stanley-Reisner algebra. The additive structure of the algebraic complex $R^{*}(K)$ can be identified with the cellular chain complex of $\left(D^{2}, S^{1}\right)^{K}$ for a special cellular decomposition (see, for example, [1]). Thus, we have an additive isomorphism $\widetilde{H}^{*}\left(\left(D^{2}, S^{1}\right)^{K} ; \mathbb{k}\right) \cong H^{*}\left(R^{*}(K), d\right)$. However, one also has a stronger result, describing the multiplicative structure of the cohomology ring $([10],[5], 6])$.

Theorem 6.3 (Baskakov-Buchstaber-Panov). The following algebras are isomorphic:

$$
H^{*}\left(\left(D^{2}, S^{1}\right)^{K} ; \mathbb{k}\right) \cong H^{*}\left(R^{*}(K), d\right) \cong \operatorname{Tor}_{\mathbb{k}[m]}^{* * *}(\mathbb{k}[K], \mathbb{k}) .
$$

Thus, rk $H^{l}\left(\left(D^{2}, S^{1}\right)^{K} ; \mathbb{k}\right)=\sum_{-i+2 j=l} \beta^{-i, 2 j}(K)$, which shows that the bigraded betti numbers admit a topological interpretation.

Now we want to briefly describe some tools needed to work with convex polytopes. A detailed survey of basic constructions of convex geometry, together with rigorous definitions, can be found in [23] and [13].

A convex polytope is a bounded set of the form

$$
P=\left\{x \in \mathbb{R}^{n}:\left\langle a_{i}, x\right\rangle+b_{i} \geqslant 0\right\} .
$$

Here $a_{i} \in \mathbb{R}^{n}, i=1, \ldots, m$, are normal vectors to the facets of the polytope and $b_{i} \in \mathbb{R}$. We assume that the inequalities (6.1) are irredundant, i.e., each of the corresponding equalities defines a facet of the polytope:

$$
\mathcal{F}_{j}=P \cap\left\{x:\left\langle a_{j}, x\right\rangle=b_{j}\right\} .
$$

We also assume that the dimension $n$ of a polytope coincides with the dimension of the ambient euclidean space. The intersections of various facets are faces of the polytope; the poset of nonempty faces of $P$ will be denoted by $\mathcal{P}$. Two polytopes with isomorphic face posets are said to be combinatorially equivalent. A polytope $P^{n}$ is said to be simple if each of its vertices is contained in exactly $n$ facets (in this case each $i$-dimensional face is contained in $n-i$ facets). $P$ is said to be simplicial if each of its proper faces is a simplex. Each convex polytope $P$ gives rise to a dual convex polytope $Q=P^{*}$ whose proper face poset $\mathcal{Q} \backslash\{Q\}$ is isomorphic to $\mathcal{P} \backslash\{P\}$ with reversed order. The dual of a simple polytope is a simplicial polytope, and vice versa.

For a fixed presentation (6.1), consider the affine map $j_{P}: \mathbb{R}^{n} \rightarrow \mathbb{R}^{m}$ :

$$
j_{P}(x)=\left(y_{1}, \ldots, y_{m}\right), \text { where } y_{i}=\left\langle a_{i}, x\right\rangle+b_{i} .
$$

The image of the polytope under this embedding lies in the nonnegative cone $\mathbb{R}_{\geqslant}^{m}$. Moreover, $j_{P}(P)=j_{P}\left(\mathbb{R}^{n}\right) \cap \mathbb{R}_{\geqslant}^{m}$, and the map $j_{P}$ is of maximal rank. Hence, the image of 
the polytope is the intersection of an $n$-dimensional plane with that nonnegative cone. Define the plane $j_{P}\left(\mathbb{R}^{n}\right)$ by a system of $m-n$ affine equations:

$$
\begin{aligned}
& j_{P}\left(\mathbb{R}^{n}\right)=\left\{x=\left(x_{1}, \ldots, x_{m}\right) \in \mathbb{R}^{m}:\right. \\
& \left.c_{j 1} x_{1}+c_{j 2} x_{2}+\ldots+c_{j m} x_{m}=d_{j} ; j=1, \ldots, m-n\right\} .
\end{aligned}
$$

Define a space $\mathcal{Z}_{P}$ as the pullback in the following diagram:

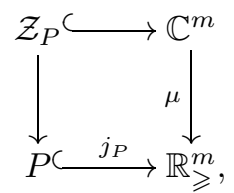

i.e., let $\mathcal{Z}_{P}=\mu^{-1}\left(j_{P}(P)\right)$. The vertical arrow denotes the moment map, which is given by $\mu:\left(z_{1}, \ldots, z_{m}\right) \mapsto\left(\left|z_{1}\right|^{2}, \ldots,\left|z_{m}\right|^{2}\right)$. The space $\mathcal{Z}_{P}$ is called the moment-angle space of $P([12,[1])$.

By (6.3), we have

$$
\begin{aligned}
& \mathcal{Z}_{P}=\left\{z=\left(z_{1}, \ldots, z_{m}\right) \in \mathbb{C}^{m}:\right. \\
& \left.\qquad c_{j 1}\left|z_{1}\right|^{2}+c_{j 2}\left|z_{2}\right|^{2}+\ldots+c_{j m}\left|z_{m}\right|^{2}=d_{j} ; j=1, \ldots, m-n\right\},
\end{aligned}
$$

i.e., the moment-angle space $\mathcal{Z}_{P}$ is the intersection of real quadrics in $\mathbb{C}^{m}$.

Theorem 6.4 (Buchstaber-Panov [12]). If $P$ is a simple polytope, then the intersection of the quadrics in (6.5) is nondegenerate. In particular, $\mathcal{Z}_{P}$ is a smooth $(m+n)$-dimensional submanifold of $\mathbb{C}^{m}$ with a trivial normal bundle.

When $P$ is a nonsimple polytope, the space $\mathcal{Z}_{P}$ has singularities. Historically, momentangle spaces of polytopes and moment-angle complexes of simplicial complexes emerged at the same time in connection with the following theorem [10, 12.

Theorem 6.5 (Buchstaber-Panov). Let $P$ be a simple polytope and $\partial P^{*}$ a simplicial decomposition of the sphere which is the boundary of the simplicial polytope dual to $P$. Then $\mathcal{Z}_{P}$ is homeomorphic to the complex $\left(D^{2}, S^{1}\right)^{\partial P^{*}}$.

Remark 6.6. The theorem also asserts that these spaces are equivariantly homeomorphic with respect to the natural action of the torus $T^{m}$ on each of them, but we will not discuss this here.

Remark 6.7. It follows from Theorems 6.5 and 6.4 that $\left(D^{2}, S^{1}\right)^{K}$ is a manifold if $K$ is the boundary of a simplicial polytope. $\left(D^{2}, S^{1}\right)^{K}$ is a topological manifold in a more general situation when $K$ is a simplicial sphere. In that case, the cohomology of $\left(D^{2}, S^{1}\right)^{K}$ has Poincaré duality. In [10], one also finds a bigraded Poincaré duality, which yields a topological interpretation of the Avramov-Golod theorem [2, [11, Theorem 3.43] on the Tor-algebra of a Gorenstein* complex.

To generalize Theorem 6.5 to arbitrary polytopes $P$, a notion of the nerve-complex of a polytope was introduced in [1].

Let $P$ be a convex polytope and $\mathcal{F}_{1}, \ldots, \mathcal{F}_{m}$ all of its facets. Then $\partial P=\bigcup_{i} \mathcal{F}_{i}$. Define the nerve-complex $K_{P}$ as a simplicial complex on the set $[m]$ whose simplices are defined by the condition $\left\{i_{1}, \ldots, i_{k}\right\} \in K_{P}$ if and only if $\mathcal{F}_{i_{1}} \cap \ldots \cap \mathcal{F}_{i_{k}} \neq \varnothing$. In other words, the simplicial complex $K_{P}$ is the nerve of the covering $\partial P=\bigcup_{i} \mathcal{F}_{i}$, which explains its name. If $P$ is a simple polytope, then $K_{P}=\partial P^{*}$.

Theorem 6.8 ([1]). Let $P$ be a convex polytope and $K_{P}$ its nerve-complex. Then $\mathcal{Z}_{P}$ is homotopy equivalent to the moment-angle complex $\left(D^{2}, S^{1}\right)^{K_{P}}$. 
Thus, the cohomology ring of $\mathcal{Z}_{P}$, viewed as the intersection of quadrics, can be computed in terms of $K_{P}$. We have $\operatorname{rk} H^{l}\left(\mathcal{Z}_{P} ; \mathbb{k}\right)=\sum_{-i+2 j=l} \beta^{-i, 2 j}\left(K_{P}\right)$. The next section deals with bigraded betti numbers of simplicial complexes of the form $K_{P}$ and of more general nerve-complexes.

\section{SPHERICAL NERVE-COMPLEXES}

The notion of a spherical nerve-complex (see the definition below) was introduced in [1. It was shown there that, for an arbitrary convex polytope $P$, the complex $K_{P}$ is a spherical nerve-complex. It was also shown that the combinatorial structure of $P$ can be recovered from that of $K_{P}$. The following problem was also posed in [1]: find necessary conditions for the simplicial complex $K$ to be the nerve-complex of some polytope. In this section, we give some new homological conditions that distinguish nerve-complexes of polytopes in the class of all spherical nerve-complexes. We shall also apply the obtained results on bigraded betti numbers of nerve-complexes of polytopes to the study of the topology of the moment-angle space $\mathcal{Z}_{P}$. First, we recall basic facts about nervecomplexes.

Let $K$ be a simplicial complex and $M(K)$ the set of its maximal by inclusion simplices. We shall say that a simplex $I \in K$ is a face simplex if it is the intersection of some number of maximal simplices: $I=J_{1} \cap \ldots \cap J_{k}, J_{i} \in M(K)$. The set of all face simplices will be denoted by $F(K)$. Then, by definition, $M(K) \subseteq F(K) \subseteq K$.

If $I \in K$ is an arbitrary simplex, then there is a unique minimal by inclusion face simplex $\bar{I}$ containing $I$. It can also be defined as the intersection of all maximal simplices of $K$ containing $I$. It is not difficult to show (see [1]) that

$$
\begin{aligned}
& \operatorname{core} \operatorname{link}_{K} I=\operatorname{link}_{K} \bar{I} \text {, } \\
& \operatorname{link}_{K} I=\operatorname{link}_{K} \bar{I} * \Delta,
\end{aligned}
$$

where $\Delta=\bar{I} \backslash I$ is the simplex on the vertices of $\bar{I}$ not contained in $I$. Thus, if $I \notin F(K)$, then $\operatorname{link}_{K} I$ is a contractible simplicial complex.

Definition 7.1. The simplicial complex $K$ is called a spherical nerve-complex of rank $n$ if the following conditions are satisfied:

(1) $\varnothing \in F(K)$ (in other words, the intersection of all maximal simplices of $K$ is empty).

(2) $F(K)$ is a graded poset of rank $n$ (this means that all of its maximal by inclusion chains contain $n+1$ elements). In this case, the rank function rank: $F(K) \rightarrow\{0,1, \ldots, n\}$ is defined, with the following properties: $\operatorname{rank}(\varnothing)=0$ and $\operatorname{rank} J=n$ for each maximal simplex $J \in M(K)$.

(3) Let $I \in F(K)$. Then the complex $\operatorname{link}_{K} I$ is homotopy equivalent to the sphere $S^{n-\operatorname{rank} I-1}$. Here we set $S^{-1}=\varnothing$.

A spherical nerve-complex $K$ is said to be reduced if each of its vertices is a face simplex: $\{v\} \in F(K)$.

Remark 7.2. If $I \in F(K)$, then $\operatorname{rank} I \leqslant|I|$. The rank of the spherical nerve-complex $K$ cannot exceed $\operatorname{dim} K+1$.

Proposition 7.3 ([1]). Let $P$ be a convex polytope of dimension $n$. Then: (1) complex $K_{P}$ is a reduced spherical nerve-complex of rank $n$; $(2)$ the set of face simplices $F\left(K_{P}\right)$, partially ordered by inclusion, is isomorphic to the poset of the nonempty faces of $P$ with reversed order.

Later, we shall prove a more general result (see Proposition [7.8). It turns out that there is a connection between the rank of a spherical nerve-complex and the depth of its Stanley-Reisner ring. 
Proposition 7.4. A spherical nerve-complex $K$ of rank $n$ is an $(n-1)$-LA-complex. Moreover, depth $\mathbb{k}[K]=n$ for any field $\mathbb{k}$.

Proof. If $I \in F(K)$, then $\operatorname{rank} I \leqslant|I|$, where $\operatorname{rank} I$ is the rank of the element $I$ of the poset $F(K)$, as in the definition of a spherical nerve-complex. The simplicial complex $\operatorname{link}_{K} I$ is homotopy equivalent to the sphere $S^{n-1-\operatorname{rank} I}$ and, therefore, is $(n-2-\operatorname{rank} I)$ acyclic. Hence, $\operatorname{link}_{K} I$ is $(n-2-|I|)$-acyclic as $n-2-\operatorname{rank} I \geqslant n-2-|I|$, according to Remark 7.2. If $I \notin F(K)$, then $\operatorname{link}_{K} I$ is contractible and hence acyclic, by (7.1). Hence, by the definition, $K$ is an $(n-1)$-LA-complex.

By Theorem 1.1, depth $\mathbb{k}[K] \geqslant n$. Notice that

$$
\beta^{-(m-n), 2 m}(K)=\operatorname{rk} \widetilde{H}^{n-1}(K ; \mathbb{k}) \neq 0,
$$

since the spherical nerve-complex $K$ is homotopy equivalent to the sphere $S^{n-1}$. Hence, $\operatorname{depth} \mathbb{k}[K]=m-\max \left\{i: \beta^{-i}(K) \neq 0\right\}=m-(m-n)=n$.

Corollary 7.5. Let $P$ be a convex polytope. Define the Stanley-Reisner algebra $\mathbb{k}[P]$ as $\mathbb{k}\left[K_{P}\right]$. Then $\operatorname{dim} P=\operatorname{depth} \mathbb{k}[P]$.

Remark 7.6. For the algebraic dimension, we have $\operatorname{dim} \mathbb{k}[P]=\operatorname{dim} \mathbb{k}\left[K_{P}\right]=\operatorname{dim} K_{P}+$ 1. Notice that, in general, $\operatorname{dim} K_{P}+1 \geqslant \operatorname{dim} P$, where equality holds if and only if $P$ is simple. Thus, $\operatorname{dim} \mathbb{k}\left[K_{P}\right] \geqslant \operatorname{depth} \mathbb{k}\left[K_{P}\right]$, which agrees with the general fact of Definition 2.1. Moreover, equality holds if and only if $P$ is a simple polytope. Only in this case is $\mathbb{k}[P]$ a Cohen-Macaulay ring, which should be expected since only in this case is $K_{P}$ a combinatorial sphere.

So far we have only learned that a spherical nerve-complex $K$ of rank $n$ on $m$ vertices is $(n-1)$-SCA, i.e., $\beta^{-i}(K)=0$ for $i>m-n$. It turns out that there are stronger properties that hold for all spherical nerve-complexes.

Proposition 7.7. Let $K$ be a spherical nerve-complex of rank $n$. Then

$$
\beta^{-(m-n), 2 m}(K)=1
$$

and $\beta^{-i, 2 j}(K)=0$ for $j-i>n$.

Proof. The equality $\beta^{-(m-n), 2 m}(K)=1$ follows directly from Hochster's formula: $\beta^{-(m-n), 2 m}(K)=\operatorname{rk} \widetilde{H}^{n-1}(K ; \mathbb{k})=1$ since $K$ is homotopy equivalent to the sphere $S^{n-1}$. The second part of the assertion is equivalent to the equality $\widetilde{H}^{i}\left(K_{\widehat{J}} ; \mathbb{k}\right)=0$ for $i \geqslant n$ and $J \subseteq[m]$. The latter can be proved by double induction: first by the rank of the nerve-complex and then by the cardinality $|J|$. Spherical nerve-complexes of rank 1 are disjoint unions of two simplices, and for them the assertion can be checked directly. If $|J|=0$, then $\widetilde{H}^{i}(K ; \mathbb{k})=0$ for $k \geqslant n$ since $K \simeq S^{n-1}$. Now let $J^{\prime}=J \sqcup\{v\}$. We prove the induction step using the Mayer-Vietoris exact sequence

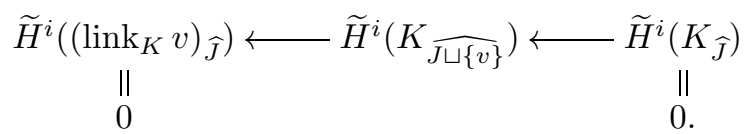

Here $\widetilde{H}^{i}\left(K_{\widehat{J}} ; \mathbb{k}\right)=0$ by the assumption of the inner induction and

$$
\widetilde{H}^{i}\left(\left(\operatorname{link}_{K} v\right)_{\widehat{J}} ; \mathbb{k}\right)=\widetilde{H}^{i}\left(\left(\operatorname{link}_{K} \overline{\{v\}}\right)_{\widehat{J}^{\prime}} *(\overline{\{v\}} \backslash\{v\})_{\widehat{J^{\prime \prime}}} ; \mathbb{k}\right)=0
$$

by the assumption of the outer induction, since $\operatorname{link}_{K} \overline{\{v\}}$ is a spherical nerve-complex of rank $n-1$. Therefore, $\widetilde{H}^{i}\left(K_{\widehat{J}\{v\}} ; \mathbb{k}\right)=0$ and the induction step is proved. 
Now we need a new construction. Let $Q^{n}$ be a convex polytope and $\mathcal{F}_{1}, \ldots, \mathcal{F}_{m}$ all of its facets. Consider an arbitrary sequence $F_{1}, \ldots, F_{r}$ of proper faces of $Q$, with repetitions allowed. We have a covering $\partial Q=\bigcup_{i} \mathcal{F}_{i} \cup \bigcup_{\alpha} F_{\alpha}$. Consider the nerve $N$ of this covering.

Proposition 7.8. In the above notation, the simplicial complex $N$ is a spherical nervecomplex of rank $n$. There is an isomorphism $\sigma$ from the face poset of $Q$ with reversed order to the poset $F(N)$ of face simplices of $N$. Moreover, for any face $F$ of the polytope $Q$, the simplicial complex $\operatorname{link}_{K} \sigma(F)$ is isomorphic to the nerve of the covering of the boundary $\partial F$ by the sets $F \cap \mathcal{F}_{i}$ and $F \cap F_{\alpha}$.

Proof. The proof is similar to that of [1, Lemma 4.7]. Denote the set of vertices of the nerve $N$ by $V$. Thus, the elements of $V$ are in one-to-one correspondence with the elements of the covering $\left\{\mathcal{F}_{i}\right\} \sqcup\left\{F_{\alpha}\right\}$. It is convenient to label the elements of the covering by the elements of $V$, i.e., $\left\{\mathcal{F}_{i}\right\} \sqcup\left\{F_{\alpha}\right\}=\left\{C_{j}\right\}$, where $j \in V$.

Let us construct a map $\sigma$ from the set $\mathcal{Q}$ of the faces of $Q$ to the set of simplices of $N$. Let $F$ be a proper face of $Q$ and let $\sigma(F)=\left\{j \in V: F \subseteq C_{j}\right\}$. Under this assignment, to the vertices $v$ of $Q$ there correspond maximal simplices $\sigma(v)$ of $N$ and vice versa: each maximal simplex of $N$ is of the form $\sigma(v)$ for some vertex $v \subset Q$. For an arbitrary face $F \in \mathcal{Q}$, we have $\sigma(F)=\bigcap_{v \in F} \sigma(v)$, i.e., $\sigma(F)$ is a face simplex. Conversely, let $I \in F(N)$; then $I=\bigcap_{s} J_{s}$, where the $J_{s} \in M(N)$ are maximal simplices. Then $J_{s}=\sigma\left(v_{s}\right)$ for vertices $v_{s}$ of $Q$. We have $I=\bigcap_{s} J_{s}=\bigcap_{s}\left\{j: v_{s} \subseteq C_{j}\right\}=\left\{j: v_{s} \subseteq C_{j}\right.$ for all $s\}=\left\{j: \bigvee_{s} v_{s} \subseteq C_{j}\right\}=\sigma\left(\bigvee_{s} v_{s}\right)$, where $\bigvee_{s} v_{s}$ denotes the smallest face of $Q$ containing all vertices $v_{s}$. Thus, the map $\sigma: \mathcal{Q} \rightarrow F(N)$ is surjective. The injectivity of this map follows from the definition.

It is not difficult to check that $\operatorname{link}_{K} \sigma(F)$ is isomorphic to the nerve of the covering of the boundary $\partial F$ by the sets $F \cap C_{j}$. A detailed proof of this assertion is given in [1].

The complexes $N$ constructed as above will be called special spherical nerve-complexes. It follows from Proposition 7.8 that the links of face simplices of a special spherical nervecomplex are themselves special spherical nerve-complexes (henceforth abbreviated as SSN-C). This observation will be needed for an induction argument in Proposition 7.10. A particular case of an SSN-C is the nerve-complex $K_{P}$ of a polytope $P$.

Lemma 7.9. Let $L$ be an $s$-LA-complex. Then $L * \Delta^{[r]}$ is an $(s+r)$-LA-complex. Here $\Delta^{[r]}$ is the simplex on the set $[r]$.

Proof. Let $V$ denote the set of vertices of $L$. By Proposition 3.2 , for any set $I \subseteq V$, the full subcomplex $L_{\widehat{I}}$ is $(s-1-|I|)$-acyclic. Consider the complex $\left(L * \Delta^{[r]}\right)_{\widehat{J}}$. If $[r] \nsubseteq J$, then this complex is isomorphic to $L_{\widehat{J} \cap V} * \Delta^{[r] \cap \widehat{J}}$ and is therefore contractible, since $[r] \cap \widehat{J} \neq \varnothing$. If $J=J^{\prime} \sqcup[r]$, then $\left(L * \Delta^{[r]}\right)_{\widehat{J}}=L_{\widehat{J}}$ is $\left(s-1-\left|J^{\prime}\right|\right)$-acyclic. Hence, $\left(L * \Delta^{[r]}\right) \widehat{J}$ is $(s+r-|J|-1)$-acyclic for all $J$, which, in view of Proposition $[3.2$, implies the desired assertion.

Proposition 7.10. Let $K$ be an SSN-C of rank $n$. Then $\beta^{-(m-n), 2 j}(K)=0$ for $j \neq m$.

Proof. By Hochster's formula, we have to show that $\widetilde{H}^{n-1-|J|}\left(K_{\widehat{J}} ; \mathbb{k}\right)=0$ for $J \neq \varnothing$. First we do the case $|J|=1$, i.e., $J=\{v\}$. Consider two cases: (1) $v \notin F(K)$ and (2) $v \in F(K)$. In the former case, we have the exact sequence from Lemma 3.3 .

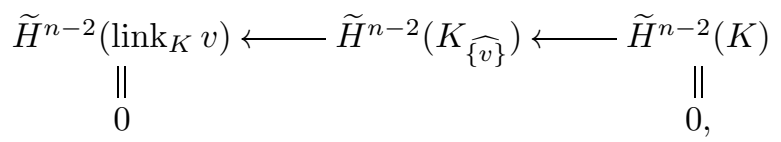

where $\operatorname{link}_{K} v$ is contractible by (7.1). This implies that $\widetilde{H}^{n-2}\left(K_{\widehat{\{v\}}} ; \mathbb{k}\right)=0$. In the latter case, by Proposition 7.8, to the vertex $v$ there corresponds a facet $\mathcal{F}$ from the covering 
$\partial Q=\bigcup \mathcal{F}_{i} \cup \bigcup F_{\alpha}$, and, moreover, this covering does not contain any additional copies of that facet. Hence, $K_{\widehat{\{v\}}}$ is the nerve of the covering $\left(\left\{\mathcal{F}_{i}\right\} \cup\left\{F_{\alpha}\right\}\right) \backslash \mathcal{F}$ of the contractible set $\partial Q \backslash \operatorname{Int}(\mathcal{F})$. Therefore, $K_{\widehat{\{v\}}}$ is acyclic.

Now consider the case $|J|>1$. Then $J=J^{\prime} \sqcup\{v\}, J^{\prime} \neq \varnothing$. Let $J^{\prime \prime}=J^{\prime} \cap V\left(\operatorname{link}_{K} v\right)$. We have an exact sequence

$$
\widetilde{H}^{n-2-\left|J^{\prime}\right|}\left(\operatorname{link}_{K_{\widehat{J}}} v\right) \longleftarrow \widetilde{H}^{n-2-\left|J^{\prime}\right|}\left(K_{\widehat{J}}\right) \longleftarrow \widetilde{H}^{n-2-\left|J^{\prime}\right|}\left(K_{\widehat{J}^{\prime}}\right)
$$

or

$$
\widetilde{H}^{n-2-\left|J^{\prime}\right|}\left(\left(\operatorname{link}_{K} v\right)_{\widehat{J^{\prime \prime}}}\right) \longleftarrow \widetilde{H}^{n-1-|J|}\left(K_{\widehat{J}}\right) \longleftarrow \widetilde{H}_{\|}^{n-2-\left|J^{\prime}\right|}\left(K_{\widehat{J}^{\prime}}\right)
$$

The equality $\widetilde{H}^{n-2-\left|J^{\prime}\right|}\left(K_{\widehat{J}^{\prime}}\right)=0$ follows from the fact that $K$ is $(n-1)$-LA. Now consider the complex $\left(\operatorname{link}_{K} v\right)_{\widehat{J^{\prime \prime}}}$. We have two cases.

(1) $v \in F(K)$. Then $\operatorname{link}_{K} v$ is SSN-C of rank $n-1$. If $J^{\prime}=J^{\prime \prime}$, then $J^{\prime \prime} \neq \varnothing$, and, therefore, by induction we have $\widetilde{H}^{i}\left(\left(\operatorname{link}_{K} v\right)_{J^{\prime \prime}}\right)=0$ for $i \leqslant n-2-\left|J^{\prime \prime}\right|$. If $\left|J^{\prime}\right|>\left|J^{\prime \prime}\right|$, then for $i \leqslant n-2-\left|J^{\prime \prime}\right|$ we have $\widetilde{H}^{i}\left(\left(\operatorname{link}_{K} v\right)_{\widehat{J^{\prime \prime}}}\right)=0$ because of the $(n-2)$-LA property for $\operatorname{link}_{K} v$.

(2) $v \notin F(K)$. Then $\operatorname{link}_{K} v=\left(\operatorname{link}_{K} \overline{\{v\}}\right) *(\overline{\{v\}} \backslash\{v\})$. The complex $\operatorname{link}_{K} \overline{\{v\}}$ is spherical of rank $n-1$, and therefore it is $(n-2)$-LA. Then the complex $\operatorname{link}_{K} v=$ $\left(\operatorname{link}_{K} \overline{\{v\}}\right) * \Delta$ is $(n-1)$-LA by Lemma 7.9 . Hence, $\widetilde{H}^{n-2-\left|J^{\prime}\right|}\left(\left(\operatorname{link}_{K} v\right)_{\widehat{J}^{\prime \prime}}\right)=0$.

In both cases, the exact sequence (17.3) implies that $\widetilde{H}^{n-1-|J|}\left(K_{\widehat{J}}\right)=0$. To finish the proof, we have to check the induction base, i.e., to prove the assertion for SSN-C of rank 1. In this case, the simplicial complex is the union of two nonempty simplices, and the assertion is true.

Bigraded betti numbers of the nerve-complexes of polytopes have some additional properties. Notice that, by the definition, a reduced special spherical nerve-complex is the nerve-complex of some polytope. Indeed, let $\left\{\mathcal{F}_{i}\right\}_{i \in[m]} \cap\left\{F_{\alpha}\right\}_{\alpha \in[r]}=\left\{C_{j}\right\}$ be a covering of the boundary $\partial Q$ of some polytope, and let $v_{\alpha}$ be the vertex of the nerve $N$ of this covering corresponding to a face $F_{\alpha}$. Then $v_{\alpha} \notin F(N)$ because $\left\{i: F \subseteq C_{i}\right\}=\overline{\{v\}} \neq\{v\}$ (each face is contained in at least one facet). Hence, if all vertices of $N$ are face vertices, then $r=0$ and $N=K_{Q}$ by definition.

Proposition 7.11. Let $P$ be a convex polytope of dimension $n$ with $m$ facets and $K_{P}$ be its nerve-complex. Then $\beta^{-i, 2 j}(K)=0$ for $j-i=n$ and $j \neq m$.

Proof. By Hochster's formula, we need to show that $\widetilde{H}^{n-1}\left(K_{J}\right)=0$ for $J \neq[m]$. Let $v \in \widehat{J}$. The complex $K_{J}$ is the nerve of the covering of $\bigcup_{i \in J} \mathcal{F}_{i}$ by the sets $\mathcal{F}_{i}$ for $i \in J$. But $\bigcup_{i \in J} \mathcal{F}_{i}$ is contained in $\bigcup_{i \in\{\widehat{v v\}}} \mathcal{F}_{i}$, which is homeomorphic to the disk $D^{n-1}$; hence its cohomology in dimension $n-1$ vanishes. The same is true for the nerve of the covering, which was claimed.

Putting together Propositions 7.4 7.7, 7.10, and 7.11, we have the following.

Theorem 7.12. Let $P^{n}$ be a polytope with $m$ facets. Then:

(1) $\beta^{-i, 2 j}\left(K_{P}\right)=0$ for $i>m-n$;

(2) $\beta^{-(m-n), 2 j}\left(K_{P}\right)=0$ for $j \neq m ; \beta^{-(m-n), 2 m}\left(K_{P}\right)=1$;

(3) $\beta^{-i, 2 j}\left(K_{P}\right)=0$ for $j-i>n$;

(4) $\beta^{-i, 2 j}\left(K_{P}\right)=0$ for $j-i=n$ and $j \neq m$. 


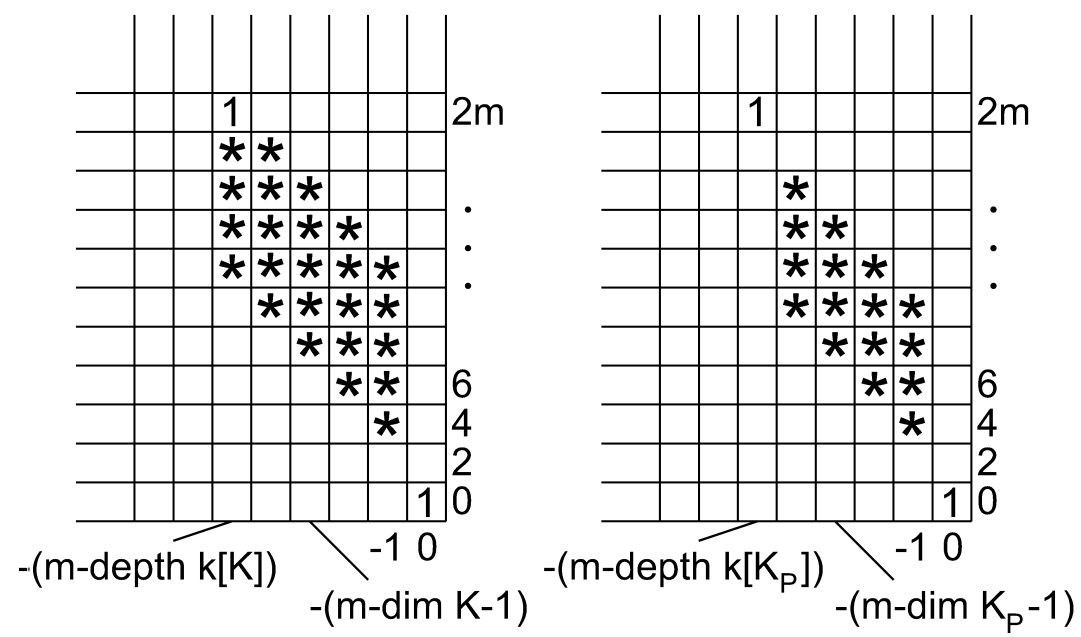

Figure 6. Bigraded betti numbers of a spherical nerve-complex (left) and of the nerve-complex of a convex polytope (right)

The distribution of bigraded betti numbers of spherical nerve-complexes and of nervecomplexes of polytopes is shown in Figure 6. One can see that for the nerve-complex of a convex polytope the distribution of bigraded betti numbers is similar to that for a Gorenstein* complex. The difference between Figures 3 and [6] is in the condition $\operatorname{depth} \mathbb{k}[K]=\operatorname{dim} \mathbb{k}[K]$, which holds for Gorenstein* complexes but not necessarily for nerve-complexes of arbitrary polytopes. Yet another distinct feature is the Poincaré duality for the $\operatorname{Tor}^{* * *}(\mathbb{k}[K] ; \mathbb{k})$-algebra when the complex $K$ is Gorenstein*, in accordance with the Avramov-Golod theorem.

We also have a topological consequence of Theorem 7.12

Proposition 7.13. Let $P^{n}$ be a polytope with $m$ facets. Then $H^{m+n}\left(\mathcal{Z}_{P} ; \mathbb{k}\right)=\mathbb{k}$ and $H^{m+n-1}\left(\mathcal{Z}_{P} ; \mathbb{k}\right)=H^{m+n-2}\left(\mathcal{Z}_{P} ; \mathbb{k}\right)=0$.

Proof. We have

$$
\operatorname{rk} H^{m+n}\left(\mathcal{Z}_{P} ; \mathbb{k}\right)=\operatorname{rk} H^{m+n}\left(\left(D^{2}, S^{1}\right)^{K_{P}} ; \mathbb{k}\right)=\sum_{-i+2 j=m+n} \beta^{-i, 2 j}\left(K_{P}\right) .
$$

Notice that, by Proposition [2.8, $\beta^{-i, 2 j}\left(K_{P}\right)=0$ for $j>m$, and when $j<m$ it follows from the condition $-i+2 j=m+n$ that $j-i>n$; for such $j$ we also have $\beta^{-i, 2 j}\left(K_{P}\right)=0$ by Theorem 7.12. Hence, the only remaining term in the sum (7.4) is $\beta^{-(m-n), 2 m}=1$.

Let us apply similar arguments to the other cohomology groups:

$$
\operatorname{rk} H^{m+n-1}\left(\mathcal{Z}_{P} ; \mathbb{k}\right)=\operatorname{rk} H^{m+n-1}\left(\left(D^{2}, S^{1}\right)^{K_{P}} ; \mathbb{k}\right)=\sum_{-i+2 j=m+n-1} \beta^{-i, 2 j}\left(K_{P}\right) .
$$

If $j \geqslant m$ and $-i+2 j=m+n-1$, then $i \geqslant m-n+1$ and, therefore, $\beta^{-i, 2 j}\left(K_{P}\right)=0$ by Theorem 7.12. If $j \leqslant m-1$ and $-i+2 j=m+n-1$, then $j-i \geqslant n$, and for such $j$ we have $\beta^{-i, 2 j}\left(K_{P}\right)=0$. All the summands on the right-hand side of (7.5) vanish.

Finally,

$$
\operatorname{rk} H^{m+n-2}\left(\mathcal{Z}_{P} ; \mathbb{k}\right)=\operatorname{rk} H^{m+n-2}\left(\left(D^{2}, S^{1}\right)^{K_{P}} ; \mathbb{k}\right)=\sum_{-i+2 j=m+n-2} \beta^{-i, 2 j}\left(K_{P}\right) .
$$

Assuming that $-i+2 j=m+n-2$, we have the following alternative. If $j \geqslant m-1$, then $i \geqslant m-n$, and equalities hold simultaneously. Both in the case of equality and in 
the opposite case, we have that $\beta^{-i, 2 j}\left(K_{P}\right)=0$. If $j \leqslant m-2$, then $j-i \geqslant n$. In this case $\beta^{-i, 2 j}\left(K_{P}\right)=0$. All summands on the right-hand side of (7.6) vanish. This finishes the proof.

Corollary 7.14. For $l=0,1,2$ we have $H^{m+n-l}\left(\mathcal{Z}_{P} ; \mathbb{k}\right) \cong H^{l}\left(\mathcal{Z}_{P} ; \mathbb{k}\right)$.

Proof. It follows from Proposition 2.8 and Hochster's formula that $H^{0}\left(\left(D^{2}, S^{1}\right)^{K}\right.$; $\mathbb{k})=\mathbb{k}$ and $H^{1}\left(\left(D^{2}, S^{1}\right)^{K} ; \mathbb{k}\right)=H^{2}\left(\left(D^{2}, S^{1}\right)^{K} ; \mathbb{k}\right)=0$ for any simplicial complex $K$. Hence, $H^{0}\left(\mathcal{Z}_{P} ; \mathbb{k}\right)=H^{0}\left(\left(D^{2}, S^{1}\right)^{K_{P}} ; \mathbb{k}\right)=\mathbb{k}$ and, similarly, $H^{1}\left(\mathcal{Z}_{P} ; \mathbb{k}\right)=$ $H^{2}\left(\mathcal{Z}_{P} ; \mathbb{k}\right)=0$.

Thus, the space $\mathcal{Z}_{P}$ has an analog of the Poincaré duality up to a certain dimension. The next example shows that, in general, for $l>2$ the duality does not hold.

Example 7.15. Consider a polytope $P=\operatorname{pyr} I^{s}$, the pyramid over a cube. In this case, $n=s+1, m=2 s+1$. By [1, Proposition 7.1], $\mathcal{Z}_{P} \cong \Sigma \Sigma\left(S^{3}\right)^{s}$, since $\mathcal{Z}_{I} \cong S^{3}$ (Example 6.2). Then $H^{3}\left(\mathcal{Z}_{P} ; \mathbb{k}\right)=0$, but $\operatorname{rk} H^{3 s-1}\left(\mathcal{Z}_{P} ; \mathbb{k}\right)=s$.

It turns out that Propositions 7.10 and 7.11 do not hold for arbitrary spherical nervecomplexes. To construct an example of a spherical nerve-complex for which these propositions fail, we need the following fact.

Proposition 7.16. Let $X$ be a polytope endowed with a regular decomposition into multiface cells such that:

(1) the dimensions of all maximal cells $C_{i}$ are $n-1$;

(2) for any two cells $A \subset B$ there is a maximal cell $C_{i}$ such that $A \subset C_{i}$, but $B \nsubseteq C_{i}$;

(3) $X \simeq S^{n-1}$

Then the nerve $N$ of the covering of $X$ by maximal cells is a spherical nerve-complex of rank $n$.

The proof of this assertion is completely analogous to the case when $X=\partial P$ (see [1] or the proof of Proposition (7.8).

Example 7.17. Figure 7 shows a polytope $X$ satisfying the conditions of Proposition 7.16. It is a two-dimensional version of Bing's house with two rooms (see 7, Ch. XIV]), with an additional hat glued on top of it. It is known that Bing's house contracts to a point. Similarly, one can show that $X$ is homotopy equivalent to the 2 -sphere. Let us decompose this space into polygons. One can see that each edge of this decomposition is contained in at least two faces, i.e., the condition of Proposition 7.16 is satisfied.

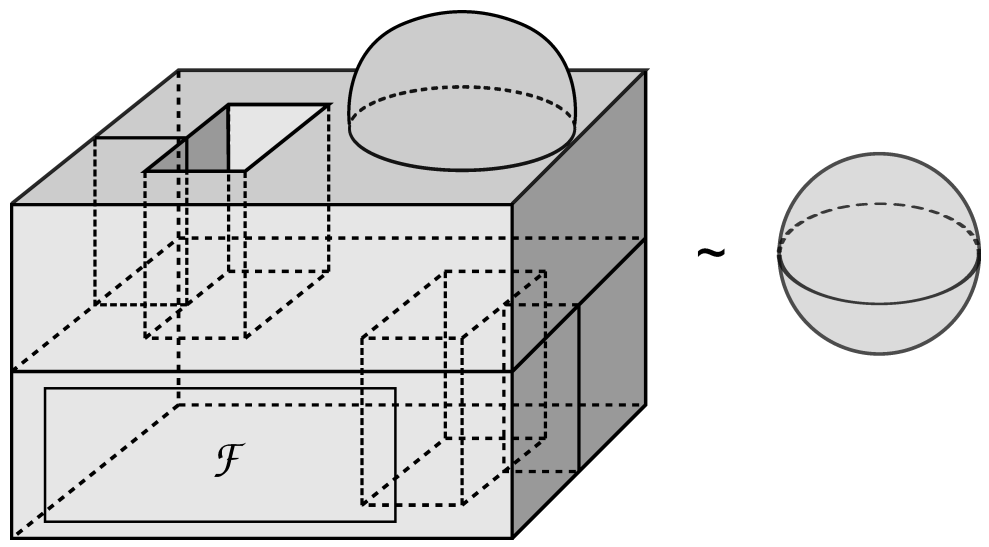

Figure 7. Polytope $X$ 


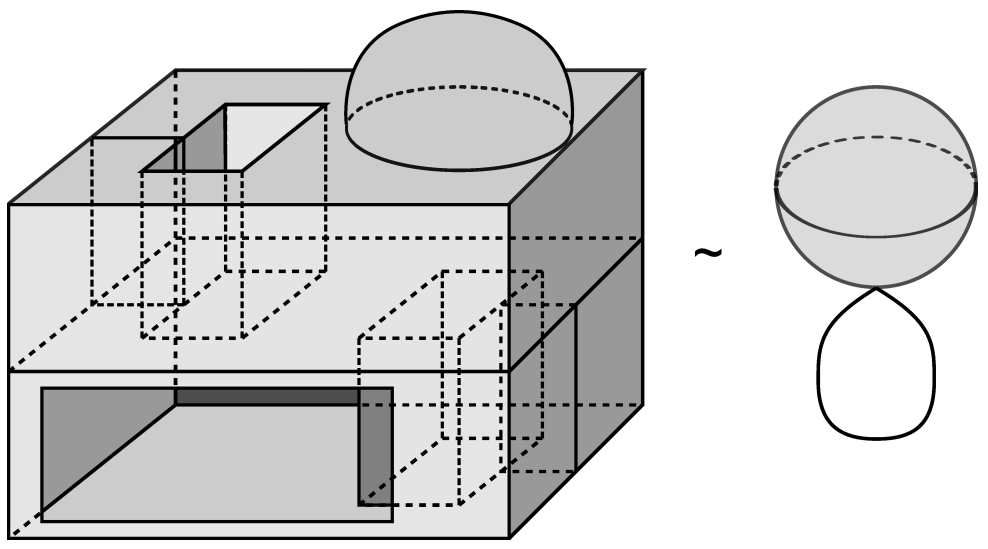

Figure 8. Polytope $X$ with face $\mathcal{F}$ removed

Hence, the nerve $K$ of the covering of $X$ by maximal cells is a spherical nerve-complex of rank 3. Let $v$ be the vertex of $K$ corresponding to the maximal cell $\mathcal{F}$ shown in the figure. Then $K_{\{\widehat{v}\}}$ is the nerve of the covering of the polytope $X \backslash \operatorname{Int}(\mathcal{F})$ shown in Figure 8 , But one can see that the "punctured" polytope $X \backslash \operatorname{Int}(\mathcal{F})$ is homotopy equivalent to the wedge sum $S^{2} \vee S^{1}$. Hence, $K_{\widehat{\{v\}}} \simeq S^{2} \vee S^{1}$ and $\widetilde{H}^{1}\left(K_{\widehat{\{v\}}} ; \mathbb{k}\right)=\widetilde{H}^{2}\left(K_{\widehat{\{v\}}} ; \mathbb{k}\right) \neq 0$. Thus, by Hochster's formula, $\beta^{-(m-3), 2(m-1)}(K) \neq 0$ and $\beta^{-(m-4), 2(m-1)}(K) \neq 0$, where $m$ is the number of vertices of $K$.

This shows that Propositions 7.10 and 7.11 cannot be generalized to the entire class of spherical nerve-complexes.

In [1, the notion of a spherical nerve-manifold was also introduced. A spherical nervemanifold is a simplicial complex $K$ such that

(1) $K$ is a spherical nerve-complex,

(2) the nerve $\operatorname{Nerv}(K)$ of the covering of the geometric realization of $|K|$ by maximal simplices is also a spherical nerve-complex.

It is known that for any convex polytope $P$ the complex $K_{P}$ is a spherical nervemanifold (in this case $\operatorname{Nerv}\left(K_{P}\right)=K_{P^{*}}$, the nerve-complex of the dual polytope). Thus, the class of spherical nerve-manifolds is smaller than the class of all spherical nervecomplexes, but it contains all complexes of the form $K_{P}$.

There is a belief that Theorem 7.12 is true for spherical nerve-manifolds. An indirect evidence in support of this conjecture is the fact that the complex $K$ from Example 7.17 is not a spherical nerve-manifold. The author is not aware of any proof of the above conjecture.

\section{ACKNOWLEDGEMEnTS}

The author expresses special thanks to his advisor Victor Matveevich Buchstaber for his constant interest in this work and for his help with the preparation of its final version. The author is truly grateful to Taras Evgenievich Panov for formulating several problems and a number of important remarks he made during discussions.

\section{REFERENCES}

[1] A. A. Aizenberg and V. M. Buchstaber, Nerve complexes and moment-angle spaces of convex polytopes, Tr. Mat. Inst. Steklova 275 (2011), 22-54. (Russian) MR2962969 
[2] L. L. Avramov and E. S. Golod, The homology of algebra of the Koszul complex of a local Gorenstein ring, Mat. Zametki 9 (1971), 53-58; English transl., Math. Notes 9 (1971), 30-32. MR0279157 $(43: 4883)$

[3] K. Baclawski, Cohen-Macaulay connectivity and geometric lattices, European J. Combin. 3 (1982), no. 4, 293-305. MR687728 (84d:06001)

[4] D. Barnette, Graph theorems for manifolds, Israel J. Math. 16 (1973), 62-72. MR0360364 (50:12814)

[5] I. V. Baskakov, Cohomology of K-powers of spaces and the combinatorics of simplicial divisions, Uspekhi Mat. Nauk 57 (2002), no. 5(347), 147-148; English transl., Russian Math. Surveys 57 (2002), no. 5, 989-990. MR1992088 (2004b:55011)

[6] I. V. Baskakov, V. M. Buchstaber, T. E. Panov, Algebras of cellular cochains, and torus actions, Uspekhi Mat. Nauk 59 (2004), no. 3(357), 159-160; English transl., Russian Math. Surveys 59 (2004), no. 3, 562-563. MR2117435(2006b:57045)

[7] R. H. Bing, The geometric topology of 3-manifolds. American Mathematical Society Colloquium Publications, 40. Amer. Math. Soc., Providence, RI, 1983. MR728227 (85j:57001)

[8] W. Bruns and J. Gubeladze, Combinatorial invariance of Stanley-Reisner rings, Georgian Math. J. 3 (1996), no. 4, 315-318. MR1397814 (97d:13025)

[9] W. Bruns and J. Herzog, Cohen-Macaulay rings, Cambridge Studies in Advanced Mathematics, 39. Cambridge University Press, Cambridge, 1993. MR,1251956 (95h:13020)

[10] V. M. Buchstaber and T. E. Panov, Actions of tori, combinatorial topology and homological algebra, Uspekhi Mat. Nauk 55 (2000), no. 5(335), 3-106; English transl., Russian Math. Surveys, 55 (2000), no. 5, 825-921. MR.1799011 (2002a:57051)

[11] V. M. Buchstaber and T. E. Panov, Torus actions in topology and combinatorics, MoskovskiưTsentr Nepreryvnogo Matematicheskogo Obrazovaniya, Moscow, 2004. (Russian)

[12] V. M. Buchstaber, T. E. Panov, N. Ray, Spaces of polytopes and cobordism of quasitoric manifolds, Mosc. Math. J. 7 (2007), no. 2, 219-242, 350. MR2337880(2008f:55006)

[13] B. Grünbaum, Convex polytopes, Second edition, Graduate Texts in Mathematics, 221. SpringerVerlag, New York, 2003. MR1976856(2004b:52001)

[14] H. Haghighi, S. Yassemi, R. Zaare-Nahandi, A generalization of $k$-Cohen-Macaulay complexes, 2009. Preprint arXiv:0912.4097v1

[15] T. Hibi Level rings and algebras with straightening laws, J. Algebra 117 (1988), no. 2, $343-362$. MR 957445 (89g:13010)

[16] T. Hibi and N. Terai, Finite free resolutions and 1-skeletons of simplicial complexes, J. Algebraic Combin. 6 (1997), no. 1, 89-93. MR 1431826 (97k:13017)

[17] M. Hochster, Cohen-Macaulay rings, combinatorics, and simplicial complexes, Ring theory, II (Proc. Second Conf., Univ. Oklahoma, Norman, Okla., 1975), pp. 171-223. Lecture Notes in Pure and Appl. Math., Vol. 26, Dekker, New York, 1977. MR0441987 (56:376)

[18] F. S. Macaulay Some properties of enumeration on the theory of modular systems, Proc. London Math. Soc. 26 (1927), no. 1, 531-555.

[19] M. Miyazaki, On 2-Buchsbaum complexes, J. Math. Kyoto Univ. 30 (1990), no. 3, $367-392$ MR $1075292(92 \mathrm{a}: 52017)$

[20] J. R. Munkres, Topological results in combinatorics, Michigan Math. J. 31 (1984), no. 1, $113-128$. MR736476 (85k:13022)

[21] G. Reisner, Cohen-Macaulay quotients of polynomial rings, Advances in Math. 21 (1976), no. 1, 30-49. MR0407036 (53:10819)

[22] R. Stanley, Combinatorics and commutative algebra, Second edition. Progress in Mathematics, 41. Birkhäuser Boston, Inc., Boston, MA, 1996. MR1453579 (98h:05001)

[23] G. M. Ziegler, Lectures on Polytopes, Graduate Texts in Mathematics, 152. Springer-Verlag, New York, 1995. MR1311028 (96a:52011)

M. V. Lomonosov Moscow State University

E-mail address: ayzenberga@gmail.com 